\title{
The Link between Integrability, Level Crossings, and Exact Solution in Quantum Models
}

\author{
H. K. Owusu, K. Wagh, and E. A. Yuzbashyan \\ Center for Materials Theory, Department of Physics and Astronomy, \\ Rutgers University, Piscataway, NJ 08854, USA
}

(Dated: October 24, 2018)

\begin{abstract}
We investigate the connection between energy level crossings in integrable systems and their integrability, i.e. the existence of a set of non-trivial integrals of motion. In particular, we consider a general quantum Hamiltonian linear in the coupling $u, H(u)=T+u V$, and require that it have the maximum possible number of nontrivial commuting partners also linear in $u$. We demonstrate how this commutation requirement alone leads to: 1) an exact solution for the energy spectrum and 2) level crossings, which are always present in these Hamiltonians in violation of the Wigner-von Neumann non-crossing rule. Moreover, we construct these Hamiltonians explicitly by resolving the above commutation requirement and show their equivalence to a sector of Gaudin magnets (central spin Hamiltonians). By contrast, fewer than the maximum number of conservation laws does not guarantee level crossings.
\end{abstract}


Contents

I. Introduction

II. The Parametrization of maximally commuting Hamiltonians

III. Algebraic properties of maximal Hamiltonians and an upper bound on the number of level crossings

IV. Mapping to the Gaudin magnets

V. Exact solution for the spectra of maximal Hamiltonians

VI. Level crossings

VII. Submaximal Hamiltonians

VIII. Summary and open questions

IX. Acknowledgements

A. Shastry's construction of commuting matrices

References 


\section{INTRODUCTION}

Level crossings - the emergence of degeneracies in a physical system at a certain value of some tuned system coupling - underly a myriad of compelling phenomena, including anomalies in relaxation rates [1], the onset of quantum chaos [2], quantum phase transitions [3], Berry's phase [4, [5] etc. It is widely believed that these degeneracies can often be understood in terms of a certain underlying symmetry. However, in many cases this connection between symmetry and degeneracy remains mysterious. This is especially true for quantum integrable systems, e.g. the 1d Hubbard, anisotropic Heisenberg, reduced BCS models etc. These systems are long known to display an abundance of level crossings [6 9], see Fig. 1, in violation of the famous Wigner-von Neumann non-crossing rule[10 16] and with no convincing symmetry explanation.

In this paper we derive the existence of level crossings and an exact solution for a general parameter-dependent quantum Hamiltonian from its integrability. Our work has been inspired in part by Refs. 17 and 18 and especially Shastry's paper [19], which opened up a new, purely algebraic perspective on quantum integrable models independent of Bethe's Ansatz. In Hamiltonian mechanics the integrability of a system with $n$ degrees of freedom is usually understood as the existence of a maximum number $(n)$ of Poisson commuting independent invariants. Then, a wellknown theorem due to Liouville and Arnold guarantees that the equations of motion can be solved by quadratures [20]. There is no similarly accepted notion of quantum integrability, especially in finite dimensional systems, e.g. discrete lattice models in condensed matter physics where the state space is generally finite. In particular, it is often unclear what constitutes an independent integral and what is the natural notion of the number of degrees of freedom. Nevertheless, it turns out that these difficulties can be circumvented if one restricts the manner in which the integrals of motion depend on the coupling.

For concreteness, let us consider Hamiltonians linear in the coupling $u$. As we are interested in discrete energy spectra, we assume that the Hamiltonian can be represented by an $N \times N$ matrix. Following the classical notion of integrability, we require the existence of the maximum possible number of independent (see below) mutually commuting integrals, $\left[H^{i}(u), H^{j}(u)\right]=0$, where $H^{i}(u)=T^{i}+u V^{i}$ are Hermitian operators. One of them is the Hamiltonian itself, e.g. $H^{1}(u) \equiv H(u)$. Using this commutation requirement alone, we derive an exact solution for the spectrum of each $H^{i}(u)$, which can be viewed as an extension of the Liouville-Arnold theorem to quantum Hamiltonians. Moreover, we are able to demonstrate that the eigenvalues of $H^{i}(u)$ are necessarily degenerate at a discrete set of values of $u$. 
First, we solve the nonlinear commutation relations $\left[H^{i}(u), H^{j}(u)\right]=0$ to obtain each $H^{i}(u)$ explicitly, see below. Interestingly, it turns out that these maximally commuting (or simply maximal) operators $H^{i}(u)$ can be mapped to exactly solvable Gaudin magnets [17, 21] (central spin Hamiltonians). The latter describe a localized spin in a magnetic field $B=u$ interacting with $N-1$ "environmental" spins and have a variety of physical applications [22 27]. The mapping to Gaudin magnets allows us to obtain the exact solution for the eigenvalues and eigenfunctions of $H^{i}(u)$ and analyze its level crossings. The total number of pairwise crossings varies as $M_{c}=(N-1)(N-2) / 2-2 K>0$, where $K$ is a positive integer such that $M_{c} \geq 1$. For instance, $N=5$ maximal operators display 2,4 , and 6 instances of level crossings. On the other hand, we demonstrate that Hamiltonians having less than the maximum number of $u$-dependent integrals can have no level crossings. Further, we show that the coupling-dependent commuting matrices obtained within the approach developed by Shastry in Ref. 19 (where it was also conjectured that these matrices always have crossings, see also Ref. 18) are maximal, even though our constructions are quite different.

Pairwise crossings of energy levels are usually understood in the context of the Wigner-von Neumann non-crossing rule. This rule initially suggested by Hund[10] and justified by Wigner and von Neumann [11] has thereafter seen restatements and refinements by a number of authors [12 16]. It states that eigenstates of the same symmetry do not cross as a function of a single coupling parameter. This can be seen, for example, from the following argument. Suppose two energy levels $E_{1}(u)$ and $E_{2}(u)$ of $H(u)$ come close at a certain $u=u_{0}$. Expanding in a vicinity of $u_{0}$ : $H(u) \approx H\left(u_{0}\right)+\left(u-u_{0}\right) V\left(u_{0}\right)$ and using ordinary perturbation theory, we obtain [28]

$$
\frac{d^{2} \Delta}{d u_{0}^{2}}=\frac{4 V_{12}^{2}\left(u_{0}\right)}{\Delta\left(u_{0}\right)}+F\left(u_{0}\right)
$$

where $\Delta\left(u_{0}\right)=E_{1}\left(u_{0}\right)-E_{2}\left(u_{0}\right), V_{12}\left(u_{0}\right)$ is the matrix element of the perturbation $V\left(u_{0}\right)$ between states $|1\rangle$ and $|2\rangle$, and $F\left(u_{0}\right)$ represents the contribution of the remaining states. We see from Eq. (11) that as the two levels approach, $\Delta\left(u_{0}\right) \rightarrow 0$, infinite repulsion sets in, preventing them from crossing. This is indeed what takes place in the absence of symmetry - energy levels repel see Fig. 2. The situation changes if the Hamiltonian $H(u)$ possesses a $u$-independent symmetry $S$, i.e. $[H(u), S]=0$. This can be a spatial rotation, translational invariance, internal space reconfiguration, etc. Because $S$ does not depend on the coupling $u$, it commutes with $H\left(u_{0}\right)$ and $V\left(u_{0}\right)$ individually. Evaluating the matrix element of $\left[V\left(u_{0}\right), S\right]$ between states $|1\rangle$ and $|2\rangle$, we obtain $V_{12}\left(u_{0}\right)=0$ for any $u_{0}$ as long as $|1\rangle$ and $|2\rangle$ have different symmetry, $s_{1} \neq s_{2}$, where $S|1\rangle=s_{1}|1\rangle$ and $S|2\rangle=s_{2}|2\rangle$. Thus, while levels of different symmetry can cross, crossings of 


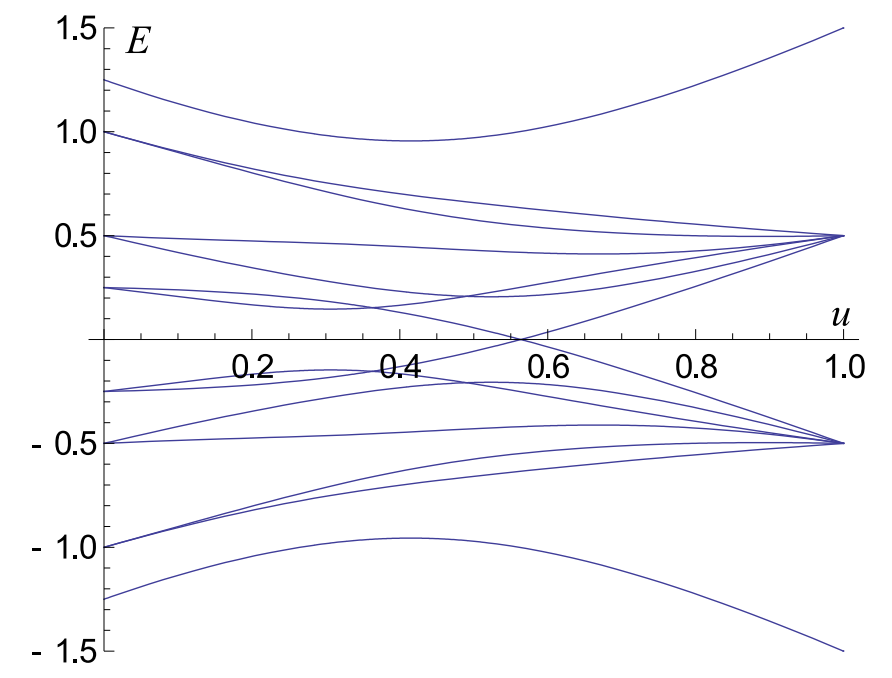

FIG. 1: Energies of 1d Hubbard model on six cites characterized by a complete set of quantum numbers, i.e. all levels have the same $u$-independent symmetry, see Refs. 6 and 18 . The energies are in units of $U-4 T$ plotted as functions of $u=U /(U-4 T)$, where $U$ is the strength of the Coulomb repulsion and $T<0$ is the hopping matrix element. The parameter $u$ varies from 0 to 1 as $U$ goes from 0 to $\infty$. Note that, in violation of the Wigner-von Neumann non-crossing rule, we see a profusion of level crossings for states of the same symmetry.

levels of the same symmetry are prohibited.

Unfortunately, this basic argument does not extend to quantum integrable Hamiltonians $H(u)$, which typically violate the non-crossing rule. Indeed, these systems show crossings of energy levels that have the same quantum numbers for all $u$-independent symmetry [6 [9], see e.g. Fig. [1, Integrable Hamiltonians are known to have special coupling dependent conserved currents, "dynamical symmetries", in addition to $u$-independent symmetries. It is tempting to attribute these crossings to such symmetries. On the other hand, it is crucial for the validity of the non-crossing rule that the symmetry $S$ be $u$-independent. Indeed, consider an integrable Hamiltonian acting on a finite-dimensional space, e.g. a lattice model with a finite number of sites. Let $H(u)$ be one of its blocks characterized by the same quantum numbers for a complete set of mutually commuting $u$-independent symmetries and let $\widetilde{H}(u)$ be the corresponding block of one of the conserved currents

$$
[H(u), \widetilde{H}(u)]=0 \quad \text { for all } u \text {. }
$$

Due to the $u$-dependence, $\widetilde{H}\left(u_{0}\right)$ no longer commutes with $V\left(u_{0}\right)$ separately and, therefore, the above argument lifting the level repulsion does not hold. At the same time, given a crossing one can always artificially engineer a "conserved current" that commutes with $H(u)$. Therefore, 


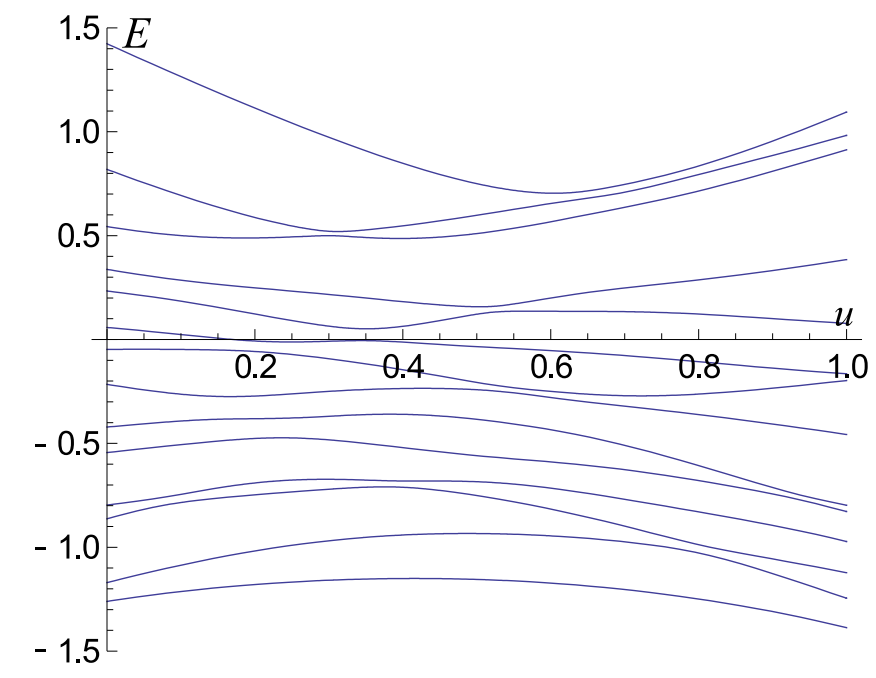

FIG. 2: Energy levels of a $14 \times 14$ Hamiltonian $H(u)=A+u B$, where independent matrix elements of $A$ and $B$ are uniformly distributed random numbers. Note that though levels do approach one another closely, they never cross. A typical Hamiltonian with no $u$-independent symmetry exhibits such level repulsion, see Eq. (11), in contradistinction to what takes place in integrable systems, see Fig. 1. Here and throughout this paper we use Mathematica program to perform numerical tests and plot the results.

restrictions on the form of $\widetilde{H}(u)$ are necessary to make meaningful contact between symmetries and degeneracies.

To be specific, let $H(u)=T+u V$ and $\widetilde{H}(u)=\widetilde{T}+u \widetilde{V}$ be Hermitian operators acting on an $N$-dimensional space, i.e. they can be represented by $N \times N$ matrices. Eq. (2) implies

$$
[T, \widetilde{T}]=[V, \widetilde{V}]=0, \quad[T, \widetilde{V}]=[\widetilde{T}, V] .
$$

For any linear $H(u)$ there are always trivially related commuting partners $\widetilde{H}(u)=a H(u)+$ $(b+c u) I$, where $I$ is an identity matrix. However, the requirement that Eq. (3) have nontrivial solutions leads to a set of nonlinear constraints that severely restrict the matrix elements of both $H(u)$ and $\widetilde{H}(u)$. For example, for $N=3$ eliminating $\widetilde{T}$ and $\widetilde{V}$ from Eq. (3), one obtains a single nonlinear constraint on the matrix elements of $H(u)$ [18]. In view of the preceding discussion regarding the prevalence of level crossings in integrable models, a natural question is whether these constraints, i.e. the existence of a nontrivial $\widetilde{H}(u)$, imply crossings in the spectrum of $H(u)$ and vice versa. This is indeed the case for $N=3$. Specifically, one can show that $3 \times 3$ matrices $H(u)=T+u V$ that have nontrivial commuting partners also have a level crossing and vice versa [18]. However, this is no longer true for $N \geq 4-$ Eq. (3) does not necessarily lead to level crossings. Moreover, crossings occur even in the absence of nontrivial partners and $u$-independent symmetries, see below. We see that a single dynamical symmetry is insufficient to explain level 
crossings. On the other hand, quantum integrable Hamiltonians typically have more than one coupling dependent commuting operator. In fact, as we show below, the maximum possible number - which turns out to be $N$ - of integrals are necessary to ensure level crossings.

We define the set of maximally commuting Hamiltonians as a vector space, $\mathcal{M}$, formed by $N \geq 3$ Hermitian, mutually commuting $N \times N$ matrices $H^{i}(u)=T^{i}+u V^{i}$ together with the $N \times N$ identity matrix $I$,

$$
\left[H^{i}(u), H^{j}(u)\right]=0, \text { for all } u \text { and } i, j=1, \ldots, N
$$

where $u$ is a real parameter. Operators $H^{i}(u)$ are assumed to be independent in that matrices $V^{i}$ are linearly independent, i.e. $\sum_{i=1}^{N} c_{i} V^{i}=0$ iff $c_{i}=0$ for all $i$ (equivalently one can require that $T^{i}$ be linearly independent). In addition, $H^{i}(u)$ are taken to have no $u$-independent symmetry common to all $H^{i}(u)[30]$,

$$
\nexists \Omega \neq a I \text { such that }\left[\Omega, H^{i}(u)\right]=0 \text { for all } u \text { and } i \text {. }
$$

Therefore, an arbitrary element $H(u)=T+u V$ of the vector space $\mathcal{M}$ has the form

$$
H(u)=\sum_{i=1}^{N} d_{i} H^{i}(u)+a I,
$$

where $d_{i}$ and $a$ are real numbers. The addition of multiples of the identity affects neither commutation relations nor level crossings of $H(u)$ and we will often omit the term $a I$ in Eq. (6) . Note also that Eq. (6) implies that operators $H^{i}(u)$ together with $I$ provide a basis in the vector space $\mathcal{M}$ of maximal Hamiltonians.

The set $\mathcal{M}$ is maximal in the sense that any Hermitian $H(u)=T+u V$ that commutes with all $H^{i}(u)$ can be written in the form (6) . Indeed, since $V$ and all $V^{i}$ mutually commute, see Eq. (3), we can go to their common eigenbasis. In this basis, the $N$ diagonal matrices $V^{i}$ are $N$ linearly independent $N$-dimensional vectors and, therefore, there exist real numbers $d_{i}$ such that $V=\sum_{i=1}^{N} d_{i} V^{i}$. The matrix $H(u)-\sum_{i=1}^{N} d_{i} H^{i}(u)$ is $u$-independent and, since it also commutes with all $H^{i}(u)$, it must be of the form $a I$ according to Eq. (5) . Thus, $H(u)$ is of the form (6). By a similar argument one can show that one of the basic matrices $H^{i}(u)$ can be chosen as $H^{i}(u)=(a+u b) I$ with real coefficients $a$ and $b$. We see that there are $N-1$ nontrivial independent commuting operators. Therefore, the first nontrivial dimensionality is $N=3$.

In what follows we begin with the explicit construction of a general, maximally commuting Hamiltonian $H(u)$. This is done in Sec. II by choosing a convenient basis in the vector space $\mathcal{M}$ and solving Eq. (44). In Sec. III we establish some useful algebraic properties of $H(u)$. Interestingly, 
it turns out that the product of any two maximally commuting Hamiltonians can be written as a linear superposition of such Hamiltonians, i.e. the set $\mathcal{M}$ has a certain quasi-ring structure.

Our parametrization of the maximally commuting Hamiltonians makes it transparent that they are related to the Gaudin magnets [17, 21], see Sec. IV] The latter are $N$ quantum spin Hamiltonians

$$
\hat{h}^{i}(B)=-B \hat{s}_{i}^{z}+\sum_{k=1}^{N} \frac{\hat{\vec{s}}_{i} \cdot \hat{\vec{s}}_{k}}{\varepsilon_{i}-\varepsilon_{k}}, \quad i=1, \ldots, N
$$

where the prime indicates that the summation is over $k \neq i, B$ is the $z$-aligned magnetic field, $\hat{\vec{s}}_{i}$ is an operator of spin of magnitude $s_{i}$, and $1 /\left(\varepsilon_{i}-\varepsilon_{k}\right)$ is the coupling between spins $\hat{\vec{s}}_{i}$ and $\hat{\vec{s}}_{k}$. The Hamiltonians $\hat{h}^{i}$ form a mutually commuting family

$$
\left[\hat{h}^{i}(B), \hat{h}^{j}(B)\right]=0 \quad \text { for any } B
$$

Note also that each $\hat{h}^{i}(B)$ is invariant under rotations around the $z$ axis, which means the $z$ component of the total spin $\hat{J}^{z}=\sum_{i=1}^{N} \hat{s}_{i}^{z}$ is conserved

$$
\left[\hat{h}^{i}, \hat{J}^{z}\right]=0, \quad i=1, \ldots, N
$$

As we will see in Sec. IV, the maximally commuting Hamiltonians (6) correspond to the sector of Gaudin magnets with $J^{z}=J_{\max }^{z}-1$, where $J_{\max }^{z}=\sum_{i=1}^{N} s_{i}$ is the maximum eigenvalue of $\hat{J}^{z}$.

In Sec. $\mathrm{V}$ we employ the mapping to the Gaudin magnets to obtain the exact solution for the spectra of maximally commuting Hamiltonians. Using this solution, we analyze the asymptotic behavior of the eigenstates in the limits $u \rightarrow \pm \infty$ in Sec. VI. Matching the two limits allows us to establish the presence of level crossings and to count them. Next, we consider Hamiltonians having less than the maximum number of commuting partners. In Sec. VII, we construct a family of $4 \times 4$ Hermitian operators linear in the coupling $u$ that have a single nontrivial partner as opposed to two partners for the $N=4$ maximal set. It turns out that these submaximal Hamiltonians often display no degeneracies at all. Finally, in Appendix we review Shastry's approach to constructing commuting real symmetric operators and show that the resulting operators are always maximal.

\section{THE PARAMETRIZATION OF MAXIMALLY COMMUTING HAMILTONIANS}

We begin our analysis by choosing a convenient basis in the vector space of maximally commuting Hamiltonians, which allows us to solve Eq. (4) explicitly. The solution yields a convenient parametrization for a general maximal Hamiltonian (6) and elucidates the algebraic structure of 
these operators. It also makes transparent the relationship between these operators and Gaudin magnets (7).

Consider the maximal operators $H^{i}(u)=T^{i}+u V^{i}$ defined in Eqs. (4) and (5). It follows from Eq. (41) that all $V^{i}$ commute with each other, see Eqs. (3) and (22). By a $u$-independent unitary transformation we go to a basis where all $V^{i}$ are diagonal. Since $V^{i}$ are also linearly independent, we can bring them to the following "canonical" form by a linear transformation

$$
D^{k}=\sum_{i} v_{i}^{k} V^{i}
$$

where $v_{i}^{k}$ are real numbers, $D^{k}$ are diagonal with a single nonzero matrix element $\left[D^{k}\right]_{j j} \equiv D_{j}^{k}=\delta_{j k}$. Next, we introduce a "canonical" basis in the space of maximally commuting operators

$$
h^{i}(u)=E^{i}+u D^{i}=\sum_{j=1}^{N} v_{j}^{i} H^{j}(u), \quad i=1, \ldots, N .
$$

The operators $h^{i}(u)$ have all the properties of maximally commuting Hamiltonians defined in Eqs. (44) and (5) as long as $H^{i}(u)$ do. In particular,

$$
\left[h^{i}(u), h^{j}(u)\right]=0, \quad \text { for all } u \text { and } i, j=1, \ldots, N \text {. }
$$

It follows from Eq. (6) that a general maximally commuting operator can be written as

$$
H(u) \equiv T+u V=\sum_{k=1}^{N} d_{k} h^{k}(u)+a I,
$$

where $d_{k}$ and $a$ are real numbers. Note that with our choice of $D^{k}, d_{k}$ are the eigenvalues of $V$.

To determine $H(u)$ explicitly, we need to solve Eq. (12). In terms of $D^{i}$ and $E^{i}$ these equations read

$$
\left[D^{i}, D^{j}\right]=0, \quad\left[D^{i}, E^{j}\right]=\left[D^{j}, E^{i}\right], \quad\left[E^{i}, E^{j}\right]=0 .
$$

The first equation holds since $D^{i}$ are diagonal. The second equation in terms of matrix elements is

$$
\left(D_{m}^{i}-D_{n}^{i}\right) E_{m n}^{j}=\left(D_{m}^{j}-D_{n}^{j}\right) E_{m n}^{i}
$$

where $E_{m n}^{j}$ is the $m n^{\text {th }}$ matrix element of $E^{j}$. By construction, the only nonzero matrix element of $D^{i}$ is $D_{i}^{i}=1$. We see that $E_{m n}^{j}=0$ as long as $m \neq n$ and $m$ and $n$ do not equal $j$. Thus, matrix $E^{j}$ only has nonzero elements of the form $E_{j m}^{j}=\left(E_{m j}^{j}\right)^{*}$ and $E_{m m}^{j}$, where $z^{*}$ denotes the complex conjugate of $z$. Note also by setting $m=i$ and $n=j$ in Eq. (15) that $E_{i j}^{i}=-E_{i j}^{j}$ for $i \neq j$. 
It remains to solve the last equation in (14). Using the above properties of matrix elements of $E^{i}$, we rewrite this equation as

$$
\begin{aligned}
& E_{i i}^{n}-E_{m m}^{n}=\frac{E_{n m}^{m} E_{m n}^{m}}{E_{n n}^{m}-E_{i i}^{m}}, \quad i \neq m, n, \\
& E_{n i}^{n}=\frac{E_{n m}^{m} E_{m i}^{m}}{E_{n n}^{m}-E_{i i}^{m}}, \quad i \neq m, n, \\
& E_{n n}^{n}-E_{m m}^{n}=E_{m m}^{m}-E_{n n}^{m}+\sum_{j \neq m, n} \frac{E_{m j}^{m} E_{j m}^{m}}{E_{n n}^{m}-E_{j j}^{m}} .
\end{aligned}
$$

By direct computation, one can show that the following ansatz satisfies Eqs. (16):

$$
\begin{aligned}
& E_{m j}^{m}=e^{\imath\left(\theta_{m}-\theta_{j}\right)} \frac{\gamma_{m} \gamma_{j}}{\varepsilon_{m}-\varepsilon_{j}}, \\
& E_{j j}^{m}=-\frac{\gamma_{m}^{2}}{\varepsilon_{m}-\varepsilon_{j}}+\psi^{m}, \quad i \neq m, \\
& E_{m m}^{m}=-\sum_{i \neq m} \frac{\gamma_{i}^{2}}{\varepsilon_{m}-\varepsilon_{i}}+\psi^{m} .
\end{aligned}
$$

where $\gamma_{j} \neq 0, \varepsilon_{j}, \theta_{m}$, and $\psi^{m}$ are real parameters. A nonzero value of $\psi^{m}$ corresponds to an overall shift of the diagonal of $E^{m}$, which yields a (nonessential) contribution $\psi^{m} I$ to $h^{m}(u)$. Note that $\gamma_{j}=0$ also satisfies Eqs. (16) but generates matrices with block diagonal structure and, therefore, $u$-independent symmetry.

Furthermore, any solution of Eqs. (16) admits parametrization (17). To establish this, it is sufficient to show that any choice of the $3 N-2$ matrix elements $E_{m n}^{m}=\left(E_{n m}^{m}\right)^{*}$ and $E_{n n}^{m}$ for a certain $m$ compatible with Eqs. (16) corresponds to a set of $3 N+1$ real parameters, $\theta_{j}, \gamma_{j}, \varepsilon_{j}$, and $\psi^{m}$. Then, Eqs. (16) ensure that all $E^{j}$ are of the form (17). The extra three parameters are an overall scale for $\varepsilon_{j}$ and $\gamma_{j}$, a shift $\varepsilon_{i} \rightarrow \varepsilon_{i}+$ const, and a shift $\theta_{j} \rightarrow \theta_{j}+$ const, which do not affect Eqs. (17). To see the correspondence between the $E_{m n}^{m}=\left(E_{n m}^{m}\right)^{*}$ and $E_{n n}^{m}$ and the $\theta_{j}, \gamma_{j}, \varepsilon_{j}$, and $\psi^{m}$, note that Eqs. (17) yield

$$
\sum_{n \neq m} \frac{E_{m n}^{m} E_{n m}^{m}}{E_{n n}^{m}-\psi^{m}}=E_{m m}^{m}-\psi^{m},
$$

which, for given $E_{m n}^{m}=\left(E_{n m}^{m}\right)^{*}$ and $E_{n n}^{m}$, can be solved for $\psi^{m}$. In seeking a common denominator, we see that it constitutes an $N^{\text {th }}$ order polynomial

$$
\prod_{j}\left(E_{j j}^{m}-\psi^{m}\right)-\sum_{n \neq m} \prod_{j \neq m, n} E_{m n}^{m} E_{n m}^{m}\left(E_{j j}^{m}-\psi^{m}\right)=0
$$


yielding $N$ solutions $\psi^{m}$. By considering the form of matrix $E^{m}$, i.e. that all matrix elements are zero save for a row, the corresponding column, and the diagonal, we find that the left hand side of Eq. (18) is the characteristic polynomial given by $\operatorname{det}\left(E^{m}-\psi^{m} I\right)$. Consequently, all $N$ solutions of Eq. (18) are guaranteed to be real as they are the eigenvalues of an explicitly Hermitian matrix. Once $\psi^{m}$ is determined, we can calculate ratios

$$
\gamma_{j}=-e^{\imath\left(\theta_{j}-\theta_{m}\right)} \frac{E_{m j}^{m}}{E_{j j}^{m}-\psi^{m}} \quad j \neq m,
$$

where $e^{2 \imath\left(\theta_{j}-\theta_{m}\right)} \equiv E_{j m}^{m} / E_{m j}^{m}, \theta_{m}$ is arbitrary and by a choice of an overall scale we set $\gamma_{m}=1$. Lastly, letting $\varepsilon_{m}=0$ (by shifting $\varepsilon_{i}$ ), we have

$$
\varepsilon_{j}=\frac{1}{E_{j j}^{m}-\psi^{m}}
$$

Each of the $N$ solutions $\psi^{m}$ to Eq. (18) will yield a distinct set $\left\{\gamma_{j}, \epsilon_{j}\right\}$, but by construction (see Eq. (18) ) corresponds to the same set $\left\{E_{m n}^{m}, E_{n m}^{m}, E_{j j}^{m}\right\}$.

Now, consider $E^{m}$ as defined in Eq. (17). The matrix $E^{m}$ with complex matrix elements $E_{m j}^{m}=\left(E_{j m}^{m}\right)^{*}, E_{j j}^{m}$, and $E_{m m}^{m}$ is conjugate to a matrix $\widetilde{E}^{m}$, i.e.

$$
E^{m}=\Sigma \widetilde{E}^{m} \Sigma^{-1}
$$

where $\Sigma$ is a diagonal matrix with entries $\Sigma_{j j} \equiv e^{\imath \theta_{j}}=e^{\imath \theta_{m}} \sqrt{\frac{E_{j m}^{m}}{E_{m j}^{m}}},\left(\widetilde{E}_{m j}^{m}\right)^{2}=E_{m j}^{m} E_{j m}^{m}, \widetilde{E}_{j j}^{m}=E_{j j}^{m}$, and $\theta_{m}$ is an arbitrary real number. Given a Hermitian $E^{m}$, we find that $\widetilde{E}^{m}$ is necessarily real symmetric matrix and $\Sigma$ is a conjugating diagonal matrix whose matrix elements are complex phases. Thus, all Hermitian maximally commuting operators are matrix conjugate to some real symmetric such. Operator spectra are invariant under matrix conjugation and, therefore, it is convenient to henceforth limit our discussion to maximally commuting real symmetric matrices, and we do so without loss of generality.

Moreover, as noted belowEq. (17), nonzero $\psi^{i}$ contributes only a multiple of the identity, $\psi^{i} I$ to each $h^{i}(u)$, which affects neither the commutation relations nor the level crossings of $h^{i}(u)$ and their linear combinations. Henceforth, we adopt a convenient "gauge choice" $\psi^{i}=0$ for all $i=1, \ldots, N$ and $\theta_{m}=0$ for all $m=1, \ldots, N$. With this choice of $\psi^{i}$ and $\theta_{m}$, we derive from Eqs. (1117) and 
the definition of $D^{i}$ the nonzero matrix elements of basic operators $h^{i}(u)$

$$
\begin{aligned}
& {\left[h^{i}(u)\right]_{i j}=\frac{\gamma_{i} \gamma_{j}}{\varepsilon_{i}-\varepsilon_{j}}, \quad i \neq j,} \\
& {\left[h^{i}(u)\right]_{j j}=-\frac{\gamma_{i}^{2}}{\varepsilon_{i}-\varepsilon_{j}}, \quad i \neq j,} \\
& {\left[h^{i}(u)\right]_{i i}=u-\sum_{j \neq i} \frac{\gamma_{j}^{2}}{\varepsilon_{i}-\varepsilon_{j}} .}
\end{aligned}
$$

Note that

$$
\sum_{i=1}^{N} h^{i}(u)=u I .
$$

Expressions (19) for matrix elements constitute a complete, explicit solution of commutation relations (12) or equivalently (41) for maximally commuting Hamiltonians. Different choices of parameters $\gamma_{j}$ and $\varepsilon_{j}$ (factoring out overall scale of $\gamma_{j}$ and $\varepsilon_{j}$, a total shift of all $\varepsilon_{j}$, and the "gauge freedom" discussed above) yield distinct families of such Hamiltonians.

Eq. (19) also determines matrix elements[29] of a general maximally commuting operator (13)

$$
\begin{gathered}
{[H(u)]_{m n}=\gamma_{m} \gamma_{n}\left(\frac{d_{m}-d_{n}}{\varepsilon_{m}-\varepsilon_{n}}\right), \quad m \neq n,} \\
{[H(u)]_{m m}=u d_{m}-\sum_{j \neq m} \gamma_{j}^{2}\left(\frac{d_{m}-d_{j}}{\varepsilon_{m}-\varepsilon_{j}}\right)}
\end{gathered}
$$

Let us also note that a convenient approach to producing nontrivial solutions of Eq. (2) was developed by Shastry in Ref. 19. Interestingly, these solutions turn out to be essentially equivalent to the maximally commuting set constructed in this section, see Appendix for details.

\section{ALGEBRAIC PROPERTIES OF MAXIMAL HAMILTONIANS AND AN UPPER BOUND ON THE NUMBER OF LEVEL CROSSINGS}

The above parametrization makes transparent a beautiful property of maximal Hamiltonians the product of two maximal operators is itself the $u$-dependent sum of maximal operators. This property, as we demonstrate in this section, allows one to express a general maximal Hamiltonian $\widetilde{H}(u)$ as a polynomial in another such Hamiltonian $H(u)$. We employ this polynomial expansion to determine the maximum number of level crossings in the eigenvalue spectrum of $H(u)$. 
First, we express the product of two basic maximally commuting operators $h^{i}(u)$ and $h^{j}(u)$ in terms of $u$-dependent linear combinations of $h^{k}(u)$. Using Eq. (19), one can show that

$$
\begin{aligned}
& h^{i}(u) \cdot h^{j}(u)=\frac{\gamma_{j}^{2}}{\varepsilon_{i}-\varepsilon_{j}} h^{i}(u)+\frac{\gamma_{i}^{2}}{\varepsilon_{j}-\varepsilon_{i}} h^{j}(u), \quad i \neq j, \\
& h^{i}(u) \cdot h^{i}(u)=\sum_{k=1}^{N}\left[u-\sum_{m \neq k} \frac{\gamma_{m}^{2}}{\varepsilon_{k}-\varepsilon_{m}}\right] h^{k}(u) .
\end{aligned}
$$

Now consider two general maximally commuting Hamiltonians (13)

$$
H(u)=\sum_{k=1}^{N} d_{k} h^{k}(u), \quad \widetilde{H}(u)=\sum_{k=1}^{N} \widetilde{d}_{k} h^{k}(u),
$$

where without loss of generality we dropped multiples of identity in Eq. (13). From Eq. (22) we derive

$$
H(u) \cdot \widetilde{H}(u)=\sum_{k=1}^{N}\left[u d_{k} \widetilde{d}_{k}-\sum_{m \neq k} \frac{\gamma_{m}^{2}\left(d_{k}-d_{m}\right)\left(\widetilde{d}_{k}-\widetilde{d}_{m}\right)}{\varepsilon_{k}-\varepsilon_{m}}\right] h^{k}(u) .
$$

This quasi-ring structure - so called because, while the sum of maximal Hamiltonians is maximal, the product is a $u$-dependent sum of such and, therefore, not generally linear in $u$ and not strictly a maximal operator - suggests a means of representing an element of a commuting maximal family by any other, see Eq. (25) below.

A typical maximal Hamiltonian $H(u)$ can be degenerate only at discrete values of $u$. Note that the only alternative to the discrete (possibly empty) set is a permanent degeneracy - when two eigenvalues of $H(u)$ coincide at all $u$ [31]. Permanent degeneracies do not occur for a generic choice of $d_{k}$ in Eq. (23). Indeed, recall that $d_{k}$ are the eigenvalues of $V$ (see below Eq. (13)). Since the eigenvalues of $H(u)=T+u V$ tend to those of $u V$ for large $u$, the spectrum of $H(u)$ is not degenerate as long as the $d_{k}$ are distinct and $V$ is itself nondegenerate.

Consider $H(u)$ at any $u$ where it is nondegenerate. Any element of its commutant - the set of all real symmetric operators that commute with $H(u)$ - can be expressed as a polynomial in $H(u)$ of the order $N-1$, i.e.

$$
\widetilde{H}(u)=\sum_{\alpha=0}^{N-1} P_{\alpha}(u) H^{\alpha}(u)
$$

where $H^{\alpha}(u) \equiv[H(u)]^{\alpha}$ and, as we will see shortly, $P_{\alpha}(u)$ are rational functions of $u$. To see that $\widetilde{H}(u)$ can be indeed written in terms of powers of $H(u)$, consider Eq. (25) in the common eigenbasis of commuting operators $H(u)$ and $\widetilde{H}(u)$ at a given $u$. Since eigenvalues $\omega_{m}$ of $H(u)$ 
are $N$ distinct real numbers, one can always find a polynomial $R_{N-1}(\omega)=\sum_{\alpha=0}^{N-1} P_{\alpha} \omega^{\alpha}$ of order $N-1$ with $N$ real coefficients $P_{\alpha}$ so that $R_{N-1}\left(\omega_{m}\right)=\widetilde{\omega}_{m}$, where $\widetilde{\omega}_{m}$ are the eigenvalues of $\widetilde{H}(u)$. Indeed, the equations $R_{N-1}\left(\omega_{m}\right)=\widetilde{\omega}_{m}$ are linear in $P_{\alpha}$ with a nonzero determinant.

Next, we observe from Eqs. (23) and (24) that

$$
H^{\alpha}(u)=\sum_{k=1}^{N} Q_{k}^{\alpha}(u) h^{k}(u)
$$

where for $\alpha \geq 1 Q_{k}^{\alpha}(u)$ is an $\alpha-1$ order polynomial in $u$ determined by recursively applying Eq. (24) and $Q_{k}^{0}=1 / u$ as follows from Eq. (20). Plugging Eq. (26) into Eq. (25) and using the second equation in (23), we obtain

$$
\sum_{k=1}^{N} \sum_{\alpha=0}^{N-1} P_{\alpha}(u) Q_{k}^{\alpha}(u) h^{k}(u)=\sum_{k=1}^{N} \widetilde{d}_{k} h^{k}(u) .
$$

Since $h^{k}(u)$ are linearly independent at any $u \neq 0$, i.e. $\sum_{k=1}^{N} f_{k}(u) h^{k}(u)=0$ if and only if $f_{k}(u) \equiv 0$ for all $u \neq 0[32]$, Eq. (27) becomes

$$
\sum_{\alpha=0}^{N-1} P_{\alpha}(u) Q_{k}^{\alpha}(u)=\widetilde{d}_{k}, \quad k=1, \ldots, N .
$$

Note that because $Q_{k}^{\alpha}(u)$ are rational functions in $u, P_{\alpha}(u)$ are also rational functions.

Because $\widetilde{H}(u)$ is arbitrary, Eqs. (28) should have solutions for $P_{\alpha}(u)$ for any $\widetilde{d}_{k}$ as long as $H(u)$ is nondegenerate. On the other hand, solutions cease to exist if and only if $\operatorname{det}\left[Q_{k}^{\alpha}(u)\right]=0$, where $Q_{k}^{\alpha}(u)$ is regarded as the $\alpha k^{\text {th }}$ matrix element of an $N \times N$ matrix. Using the fact that $Q_{k}^{\alpha}(u)$ is a polynomial in $u$ of degree $\alpha-1$ for $\alpha \geq 1$ and $Q_{k}^{0}=1 / u$, one can show that $\operatorname{det}\left[Q_{k}^{\alpha}(u)\right]=\mathcal{P}(u) / u$, where $\mathcal{P}(u)$ is a polynomial in $u$ of order $\sum_{m=0}^{N-2} m$. The real roots of the equation $\mathcal{P}\left(u_{\gamma}\right)=0$ are the values of $u=\left\{u_{\gamma}\right\}$ where $H(u)$ is degenerate. Thus, the maximum possible number of level crossings in the eigenvalue spectrum of an $N \times N$ maximally commuting Hamiltonian is

$$
M_{c}^{\max }=\frac{(N-1)(N-2)}{2} .
$$

The polynomial $\mathcal{P}(u)$ is of real coefficients and, therefore, its complex roots come in conjugate pairs. Consequently, the number of real roots of $\mathcal{P}(u)$ falls from the maximum $M_{c}^{\max }$ in decrements of two. This enforces a parity such that the number of real roots is odd for integers of the form $4 m$, $4 m+1$ and even for integers $4 m+2,4 m+3, m \in \mathbb{N}$. Ostensibly, when real roots of $\mathcal{P}(u)$ are degenerate their number need not correspond to the number of distinct crossings. In principle, a multiple real root of $\mathcal{P}(u)$ could correspond to a single pairwise crossing. Numerically, however, we have observed that such multiplicities occur only when more than two levels cross simultaneously, i.e. at the same value of $u$. 


\section{MAPPING TO THE GAUDIN MAGNETS}

In this section, we show that maximally commuting Hamiltonians $h^{i}(u)$ are equivalent to the Gaudin magnets,

$$
\hat{h}^{i}(B)=-B \hat{s}_{i}^{z}+\sum_{k=1}^{N} \frac{\hat{\vec{s}}_{i} \cdot \hat{\vec{s}}_{k}}{\varepsilon_{i}-\varepsilon_{k}}, \quad i=1, \ldots, N,
$$

in the next to highest weight sector, $J^{z}=J_{\max }^{z}-1$, where $J^{z}$ is the $z$ projection of the total spin, $\hat{\vec{s}}_{i}=\left\{\hat{s}_{i}^{x}, \hat{s}_{i}^{y}, \hat{s}_{i}^{z}\right\}$ is the $i^{\text {th }}$ spin 3 -vector of magnitude $s_{i}$ and $\left[\hat{s}_{i}^{\alpha}, \hat{s}_{j}^{\beta}\right]=\epsilon_{\alpha \beta \gamma} \hat{s}_{i}^{\gamma} \delta_{i j}$. This mapping is very useful as Gaudin magnets (central spin Hamiltonians) have been extensively studied [17, 21, 33, 34, 35, 36, 37]. For example, an exact solution for the eigenstates and eigenvalues is available 17, 21]. We employ it in subsequent sections to obtain the spectra of maximally commuting Hamiltonians and to analyze their level crossings. This mapping also implies that all our conclusions regarding maximal Hamiltonians, e.g. the presence and the number of level crossings, quasi-ring structure (22) etc. can be immediately transferred to the corresponding sector of Gaudin magnets and their derivative models, such as the reduced BCS model[38, 39, 40]. At the same time, other sectors of the Gaudin model as well as more general models [36] of which it is a particular case can provide examples of Hamiltonians with less then the maximum number of commuting partners.

Since Gaudin magnets (30) commute with the $z$ projection of the total spin $\hat{J}^{z}$, see Eq. (9), they are block-diagonal in any basis where $\hat{J}^{z}$ has a definite value. Different blocks can be labeled by the eigenvalues of $\hat{J}^{z}$. Consider the sector $J^{z}=J_{\max }^{z}-1$, where $J_{\max }^{z}=\sum_{i=1}^{N} s_{i}$ is the maximum eigenvalue of $\hat{J}^{z}$. It is populated by $N$ basic states

$$
|k\rangle=\frac{\hat{s}_{k}^{-}|0\rangle}{\sqrt{2 s_{k}}}, \quad k=1, \ldots, N
$$

where $|0\rangle$ is the highest weight state $J^{z}=J_{\max }^{z}$, i.e. $\hat{s}_{k}^{+}|0\rangle=0$ for all $k$, and the highest weight $s_{k}$ for each spin $\hat{s}_{k}$ is given by $\hat{s}_{k}^{z}|0\rangle=s_{k}|0\rangle$. Therefore, Gaudin Hamiltonians (30) are $N$ commuting real symmetric $N \times N$ matrices in this sector. Since there is also no obvious $B$-independent symmetry

( $\hat{J}^{z} \propto I$ within a given sector), the $\hat{h}^{i}(B)$ appear to be good candidates for a maximally commuting set.

To check this, let us evaluate the nonvanishing matrix elements of $\hat{h}^{i}(B)$ given by Eq. (30) in 
the normalized basis (31). We obtain

$$
\begin{aligned}
& \left\langle i\left|\hat{h}^{i}(B)\right| j\right\rangle=\frac{\sqrt{s_{i} s_{j}}}{\varepsilon_{i}-\varepsilon_{j}}, \quad j \neq i, \\
& \left\langle j\left|\hat{h}^{i}(B)\right| j\right\rangle=-\frac{s_{i}}{\varepsilon_{i}-\varepsilon_{j}}+\left[-B s_{i}+\sum_{k \neq i} \frac{s_{i} s_{k}}{\varepsilon_{i}-\varepsilon_{k}}\right], \quad j \neq i, \\
& \left\langle i\left|\hat{h}^{i}(B)\right| i\right\rangle=B-\sum_{k \neq i} \frac{s_{k}}{\varepsilon_{i}-\varepsilon_{k}}+\left[-B s_{i}+\sum_{k \neq i} \frac{s_{i} s_{k}}{\varepsilon_{i}-\varepsilon_{k}}\right] .
\end{aligned}
$$

Comparing these expressions to matrix elements of $h^{i}(u)$ in Eq. (19), we observe that with the identifications $B=u$ and $s_{k}=\gamma_{k}^{2}$ the two matrices differ only by a multiple of an identity matrix $\psi^{i} I$, where

$$
\psi^{i}=-B s_{i}+\sum_{k \neq i} \frac{s_{i} s_{k}}{\varepsilon_{i}-\varepsilon_{k}}
$$

Recall that we arbitrarily selected a "gauge" $\psi^{i}=0$ for maximally commuting Hamiltonians $h^{i}(u)$, see Eq. (17) and the text above Eq. (19). This constant overall shift of all eigenvalues of $h^{i}(u)$ affects neither its eigenstates nor the degeneracies.

Thus, we see that Gaudin Hamiltonians (30) in the next to highest weight sector $J^{z}=J_{\max }^{z}-1$ are equivalent to basic maximal Hamiltonians $h^{i}(u)$ with

$$
u=B, \quad \gamma_{k}^{2}=s_{k}
$$

and vice versa. Note that the magnitudes of quantum spins, $s_{k}$, take half-integer values for finite dimensional representations of the spin $s u(2)$ algebras, while $\gamma_{k}$ are arbitrary real numbers. We believe that this restriction can be lifted by moving to an appropriate infinite dimensional representations of the $s u(2) \mathrm{s}$, where the highest weight states are still well defined but $s_{k}$ take arbitrary real values[41]. Indeed, we have verified that, at least in our sector $J^{z}=J_{\max }^{z}-1$, in all expressions

for the eigenvalues and eigenstates of $\hat{h}^{i}(B)$ (see below) the replacements $B \rightarrow u$ and $s_{k} \rightarrow \gamma_{k}^{2}$ with arbitrary real $\gamma_{k}$ produce the correct corresponding eigenvalues and eigenstates of $h^{i}(u)$.

\section{EXACT SOLUTION FOR THE SPECTRA OF MAXIMAL HAMILTONIANS}

A particularly useful consequence of the mapping (34) between Gaudin magnets $\hat{h}^{i}(B)$ and maximally commuting Hamiltonians $h^{i}(u)$ is that one can obtain the exact solution for $h^{i}(u)$ by 
importing the known exact solution for the spectra of $\hat{h}^{i}(B)$ [17, 21]. The latter has been derived both from the properties of the Gaudin algebra[17] and by Bethe's Ansatz [36].

The exact eigenvalues of the Gaudin Hamiltonian (30), $\hat{h}^{i}(B)$, in the next to highest weight sector $J^{z}=J_{\max }^{z}-1$ are

$$
\left(\lambda_{m}^{i}\right)_{G}=\frac{s_{i}}{x_{m}^{G}-\varepsilon_{i}}+\psi^{i},
$$

where $\psi^{i}$ is the overall shift of all eigenvalues given by Eq. (33) and $x_{m}^{G}$ are the solutions of the following equation:

$$
B=\sum_{k=1}^{N} \frac{s_{j}}{x_{m}^{G}-\varepsilon_{k}} .
$$

Note that if this equation is brought to the common denominator, the numerator becomes a polynomial of order $N$ in $x_{m}^{G}$. Therefore, there are $N$ solutions for $x_{m}^{G}$ and $N$ eigenvalues (35) as it should be since there are $N$ states in this sector, see Eq. (31). The unnormalized eigenstates (common to all $\left.\hat{h}^{i}(B)\right)$ corresponding to eigenvalues (35) are

$$
\left|\lambda_{m}\right\rangle_{G}=\sum_{k=1}^{N} \frac{\sqrt{s_{k}}|k\rangle}{x_{m}^{G}-\varepsilon_{k}}
$$

where the basic states $|k\rangle$ have been introduced in Eq. (31). A concise derivation of Eqs. (35 40) and (37) can be found in Refs. 17, 21, and 37 .

Using the mapping (34) between basic maximal operators $h^{i}(u)$ and Gaudin Hamiltonians, we obtain from Eq. (35) the energies of $h^{i}(u)$

$$
\lambda_{m}^{i}=\frac{\gamma_{i}^{2}}{x_{m}-\varepsilon_{i}}
$$

Note that we set the overall shift $\psi^{i}=0$ in accordance to the discussion surrounding Eq. (33). The corresponding common eigenstates of all $h^{k}(u)$ are

$$
\left|\lambda_{m}\right\rangle=\sum_{k=1}^{N} \frac{\gamma_{k}|k\rangle}{x_{m}-\varepsilon_{k}}
$$

where $|k\rangle$ now stands for a basic vector for matrices $h^{i}(u)$, i.e. its $j^{\text {th }}$ component is $|k\rangle_{j}=\delta_{j k}$. In Eqs. (38) and (37) $x_{m}$ are solutions of the following equation:

$$
u=\sum_{k=1}^{N} \frac{\gamma_{k}^{2}}{x_{m}-\varepsilon_{k}} \equiv f\left(x_{m}\right), \quad m=1, \ldots, N,
$$

which follows from Eq. (36). That Eqs. (38/39) and (40) yield the correct spectrum of $h^{i}(u)$ can be verified directly using the matrix form (19) of $h^{i}(u)$. Finally, using Eq. (23), we derive the energies 


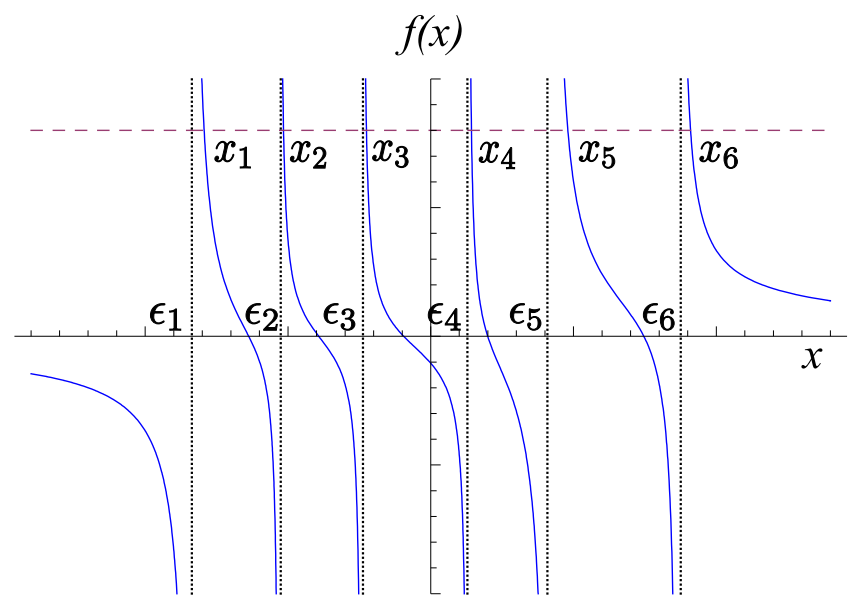

FIG. 3: A plot of $f(x)=\sum_{k=1}^{N} \gamma_{k}^{2}\left(x-\varepsilon_{k}\right)^{-1}$ for $N=6$. Solutions of the equation $f\left(x_{m}\right)=u$ determine the energies (41) and eigenstates (39) of a general maximal Hamiltonian $H(u)$. There are $N$ points, $x_{m}$ with $m=1, \ldots, N$, where $y=f(x)$ intersects $y=u$ (dashed horizontal line) yielding $N=6$ eigenstates. Note that $\varepsilon_{m}<x_{m}<\varepsilon_{m+1}$ except in the case of $x_{N}$ for which we have $\varepsilon_{N}<x_{N}$ for $u>0$ and $x_{N}<\varepsilon_{N+1} \equiv \varepsilon_{1}$ for $u<0$. Furthermore, we see that $x_{m} \rightarrow \varepsilon_{m}$ as $u \rightarrow \infty$ and $x_{m} \rightarrow \varepsilon_{m+1}$ as $u \rightarrow-\infty$. This observation allows us to determine the behavior of the energies in the $u \rightarrow \pm \infty$ limits, see Eq. (44).

of a general maximally commuting Hamiltonian, $H(u)=T+u V$,

$$
\omega_{m}=\sum_{k=1}^{N} \frac{d_{k} \gamma_{k}^{2}}{x_{m}-\varepsilon_{k}} .
$$

The corresponding eigenstates are still given by Eq. (39).

Let us analyze the flow of eigenvalues $\omega_{m}$ of $H(u)$ with $u$ and determine their behavior in the $u \rightarrow \pm \infty$ limits. Consider Eq. (40). Let $\varepsilon_{k}$ be ordered as $\varepsilon_{1}<\varepsilon_{2}<\cdots<\varepsilon_{N}$. The right hand side of Eq. (40) is plotted in Fig. 3, Note that $f(x) \rightarrow+\infty$ as $x \rightarrow \varepsilon_{k}^{+}$and $f(x) \rightarrow-\infty$ as $x \rightarrow \varepsilon_{k+1}^{-}$. It follows that the equation $u=f\left(x_{m}\right)$ has a real root between $\varepsilon_{k}$ and $\varepsilon_{k+1}$ for any $k$. Let us number the roots $x_{m}$ so that $\varepsilon_{m}<x_{m}<\varepsilon_{m+1}$. Note from Fig. 3 that for the last root $x_{N}$ we have $\varepsilon_{N}<x_{N}$ for $u>0$ and $x_{N}<\varepsilon_{N+1} \equiv \varepsilon_{1}$ for $u<0$, where from now on we identify indices $m$ and $m+N$ that differ by a multiple of $N$. Further, observe from Fig. 3 that $x_{m} \rightarrow \varepsilon_{m}$ as $u \rightarrow+\infty$. In this limit $k=m$ term dominates Eqs. (40) and (41) and we obtain $\gamma_{m}^{2} /\left(x_{m}-\varepsilon_{m}\right) \approx u$ and $\omega_{m} \approx d_{m} \gamma_{m}^{2} /\left(x_{m}-\varepsilon_{m}\right) \approx u d_{m}$. Similarly, for $u \rightarrow-\infty$ we have $x_{m} \rightarrow \varepsilon_{m+1}$ and $\omega_{m} \approx u d_{m+1}$. Therefore,

$$
\omega_{m} \rightarrow-|u| d_{m+1} \quad \text { as } u \rightarrow-\infty, \quad \omega_{m} \rightarrow|u| d_{m} \quad \text { as } u \rightarrow+\infty
$$


At this point it is convenient to rescale the Hamiltonian

$$
H^{\prime}(u)=\frac{H(u)}{\sqrt{u^{2}+1}}=\frac{T+u V}{\sqrt{u^{2}+1}} .
$$

Note that this does not affect the level crossings, i.e. $H^{\prime}(u)$ and $H(u)$ have crossings (if any) at the same values of $u$. Eq. (42) implies

$$
\omega_{m}^{\prime} \rightarrow-d_{m+1} \quad \text { as } u \rightarrow-\infty, \quad \omega_{m}^{\prime} \rightarrow d_{m} \quad \text { as } u \rightarrow+\infty
$$

where $\omega_{m}^{\prime}$ is the eigenvalue of $H^{\prime}(u)$ corresponding to the eigenstate $\left|\lambda_{m}\right\rangle$. Recall that $d_{k}$ are the eigenvalues of $V$, see the text below Eq. (13). We see from Eq. (43) that the eigenvalues of $H^{\prime}(u)$ indeed should tend to $d_{k}$ in $u \rightarrow \pm \infty$ limits consistent with Eq. (44). The latter equation however provides much more detailed information - it shows to which particular $d_{k}$ the eigenvalue corresponding to a given eigenvector tends in each limit. We will use Eq. (44) in the next section to study the crossings of energy levels of a general maximally commuting Hamiltonian $H(u)$.

\section{LEVEL CROSSINGS}

In this section, we establish the presence of energy level crossings in the spectrum of an arbitrary maximally commuting Hamiltonian $H(u)=T+u V$ (6) . This provides an explanation of the level crossing phenomenon in the absence of any $u$-independent symmetry based solely on the fact that $H(u)$ has the maximum possible number of independent commuting partners, see the text above Eq. (4). Further, we determine the number of level crossings as it depends on the ordering of the eigenvalues $d_{k}$ of the perturbation operator $V$ and argue that this number takes values

$$
M_{c}=\frac{(N-1)(N-2)}{2}-2 K, \quad K=0,1, \ldots, K_{\max },
$$

where $N$ is the dimensionality of the state space of $H(u)$ and $K_{\max }$ is the integer part (floor) of $(N-1)(N-2) / 4-1 / 2$. For example, $N=3$ maximally commuting operators have a single level crossing, while for $N=6$ we have $M_{c}=2,4,6,8$, and 10 . We also develop an approach that allows us to readily predict the minimum allowed number of crossings for a given $H(u)$ from the ordering of $d_{k}$.

Consider a Hamiltonian (not necessarily belonging to any commuting family) that depends on a real parameter $u$. Suppose $\left|n_{i}\right\rangle$ are its eigenstates and $E_{n_{1}}^{-}<E_{n_{2}}^{-}<\ldots$ are the corresponding energies at large negative $u$. There is only one way to avoid crossings - the order of eigenvalues $E_{n_{i}}^{+}$at $u \rightarrow \infty$ must be exactly the same as that at $u \rightarrow-\infty$, i.e. $E_{n_{1}}^{+}<E_{n_{2}}^{+}<\ldots$ This is what 
happens with a typical Hamiltonian in agreement with the Wigner-von Neumann non-crossing rule, Fig. 2, If, on the other hand, the relative order of any two energies changes, at least one level crossing must occur. For example, $E_{n_{1}}^{-}<E_{n_{4}}^{-}$and $E_{n_{1}}^{+}>E_{n_{4}}^{+}$means that the difference $E_{n_{1}}(u)-E_{n_{4}}(u)$ changes sign as $u$ evolves from $-\infty$ to $\infty$. By continuity this implies a crossing of levels corresponding to eigenstates $\left|n_{1}\right\rangle$ and $\left|n_{4}\right\rangle$ at a certain value of $u$. This is observed in blocks of quantum integrable Hamiltonians characterized by the same $u$-independent symmetry, see e.g. Fig. 1. Numerical spectra of maximal Hamiltonians display the same behavior, Figs. 5] and 8 8 .

Now let us turn our attention to an arbitrary maximally commuting Hamiltonian $H(u)$. In the previous section we have established the behavior of its energies in $u \rightarrow \pm \infty$ limits. It follows from Eq. (44) that the energy level of $H(u)=T+u V$ (with appropriate rescaling (43)) that starts from $-d_{k}$ at $u \rightarrow-\infty$ ends at $d_{k-1}$ at $u \rightarrow \infty$. Symbolically, this can be represented by

$$
k \longmapsto k-1, \quad(\bmod N)
$$

Note that we cannot fix an ordering of $d_{k}$ without loss of generality, as the $d_{k}$ correspond to $\varepsilon_{k}$, see e.g. Eq. (41), and we have already fixed the order of $\varepsilon_{k}$ so that $\varepsilon_{1}<\varepsilon_{2}<\cdots<\varepsilon_{N}$. First, we assume that all $d_{k}$ are distinct as is generally the case. Eq. (46) implies that the flow of energy levels from $u=-\infty$ to $u=\infty$ can be schematically depicted using the following rules:

1. Create two columns in which $\left\{-d_{k}\right\}$ and $\left\{d_{k}\right\}$ are both in descending order and replace each $d_{k}$ with its lower index $k$, i.e.

$$
\begin{gathered}
-d_{i} \\
-d_{j} \\
\vdots \\
-d_{l} \\
-d_{m}
\end{gathered}|\quad| \begin{array}{ccc}
d_{m} & & i \\
d_{l} & & j \\
\vdots & \Longrightarrow & \vdots \\
d_{j} & & l \\
d_{i} & & m
\end{array}|\quad| \begin{gathered}
m \\
\vdots \\
i
\end{gathered}
$$

2. Draw a line connecting $j$ in the left column to $j-1$ in the right. These lines represent energy levels of $H(u)$. Consequently, their crossings imply crossings of the corresponding energy levels of $H(u)$.

An example of an energy level diagram generated using the above prescription for $N=5$ is shown in Fig. 4. It corresponds to the ordering $d_{4}>d_{5}>d_{3}>d_{1}>d_{2}$ and predicts six level crossings. It also specifies which levels cross, e.g. the top level connecting 2 and 1 crosses with the next in energy 


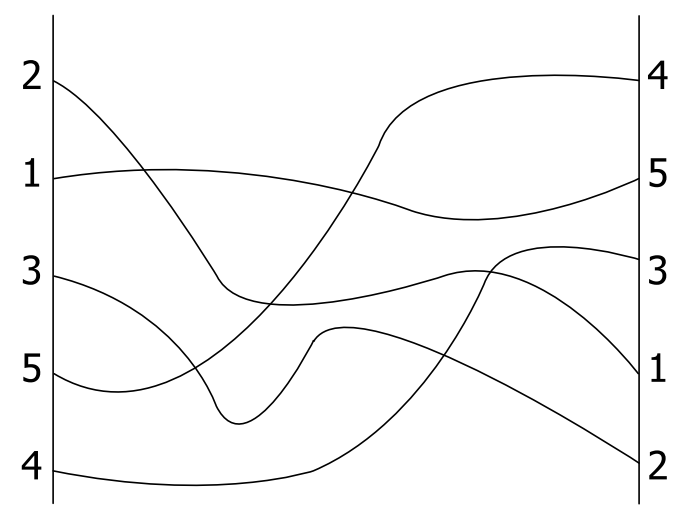

FIG. 4: Schematic energy level diagram for general $5 \times 5$ maximal Hamiltonians $H(u)=T+u V$ drawn using the rules (47). The eigenvalues $d_{k}$ of $V$ are ordered such that $d_{4}>d_{5}>d_{3}>d_{1}>d_{2}$. The diagram shows six level crossings for this ordering and specifies which levels cross, e.g. the level $4 \rightarrow 3$ first crosses $3 \rightarrow 2$ and then $2 \rightarrow 1$. Compare to Fig. 5 and note that the crossings predicted by the above diagram are exactly the same as actual numerical crossings for this ordering. Note also that according to Eq. (45) six is the maximum allowed number of crossings for this ordering of $d_{k}$ and multiple crossings of the same two levels are therefore forbidden for this ordering.

level connecting 1 and 5 . We see that the crossing predicted by Fig. 4 are exactly the same as those of actual levels of a maximally commuting operator with that ordering shown in Fig. 5, The latter has been obtained by numerical diagonalization of a $5 \times 5$ maximally commuting operator (21), $H(u)$, with randomly chosen $\gamma_{k}, \varepsilon_{k}$ and random $d_{k}$ obeying the above ordering. More examples of level diagrams are shown in Figs. 6, 7, and 9 and discussed in detail below. Next, we explore further consequences of Eq. (46).

Inevitability of level crossings for maximally commuting operators. In Sec. III we have seen that the maximum allowed number of level crossings is $(N-1)(N-2) / 2$, see Eq. (29). Now let us show that at least one crossing must be present in the spectrum of any maximally commuting $H(u)=T+u V$. Suppose the eigenvalues of $V$ are ordered as $d_{i}<d_{j}<\cdots<d_{l}<d_{m}$ as shown in the diagram (47) and assume there are no crossings. Then, the top level must go from $i$ on the left to $m$ on the right, i.e. $i \rightarrow m$, the next level starting at $j$ on the left must be connected to $l$ on the right, $j \rightarrow l$ etc. Finally, we must have $l \rightarrow j$ and $m \rightarrow i$. Consider in particular levels $i \rightarrow m$ and $m \rightarrow i$. According to Eq. (46), this asymptotic behavior implies $m=i-1(\bmod N)$ and $i=m-1(\bmod N)$. We obtain $0=2(\bmod N)$, which does not hold for any $N \geq 3$, i.e. the above assumption that levels do not cross cannot be true. Thus, we have demonstrated that at least one level crossing is always present.

The maximum number of crossings $M_{c}^{\max }=(N-1)(N-2) / 2$ is realized e.g. for the ordering 


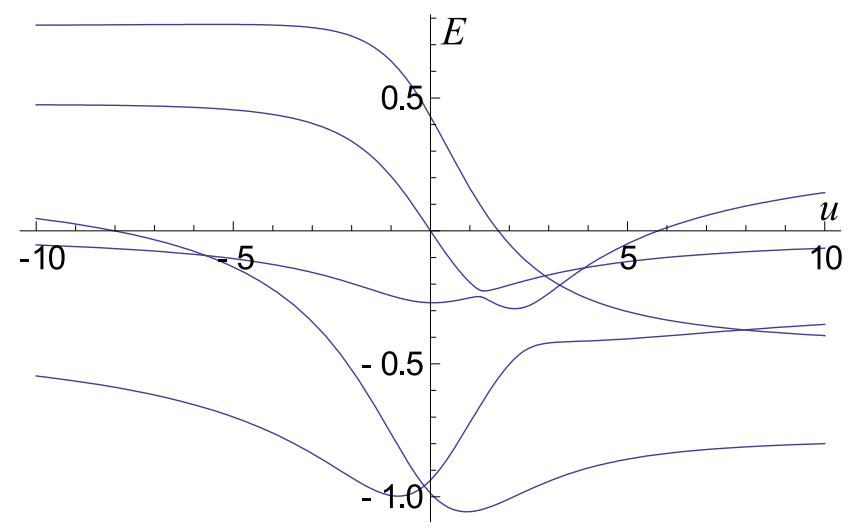

FIG. 5: Numerical energy levels of a $5 \times 5$ maximal Hamiltonian $H(u)=T+u V$ with the same ordering of eigenvalues of $V$ as that in Fig. 4. Energies are scaled with a factor $\left(u^{2}+1\right)^{-1 / 2}$ to highlight their asymptotic approach to eigenvalues of $V$. Matrix elements of $H(u)$ are generated using Eq. (21) with random $\gamma_{k}, \varepsilon_{k}$ and random $d_{k}$ constrained to obey the ordering of Fig. 4. Note that the number of crossings as well as the levels that cross are exactly the same as those predicted by Fig. 4 .

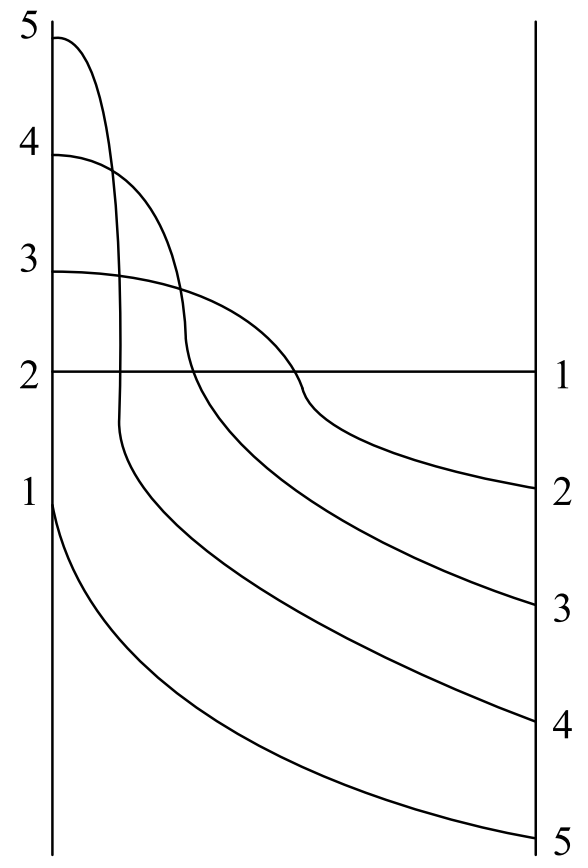

FIG. 6: A schematic diagram corresponding to maximum level crossings with $N=5$. As is evident, $N \rightarrow N-1$ line has $N-2$ crossings, $N-1 \rightarrow N-2$ adds $N-3$ new crossings and so on, till the line $3 \rightarrow 2$ adds only 1 new crossing. Thus, the maximum number of crossings, $1+2+3+\ldots+(N-2)=(N-1)(N-2) / 2$, can be confirmed. 

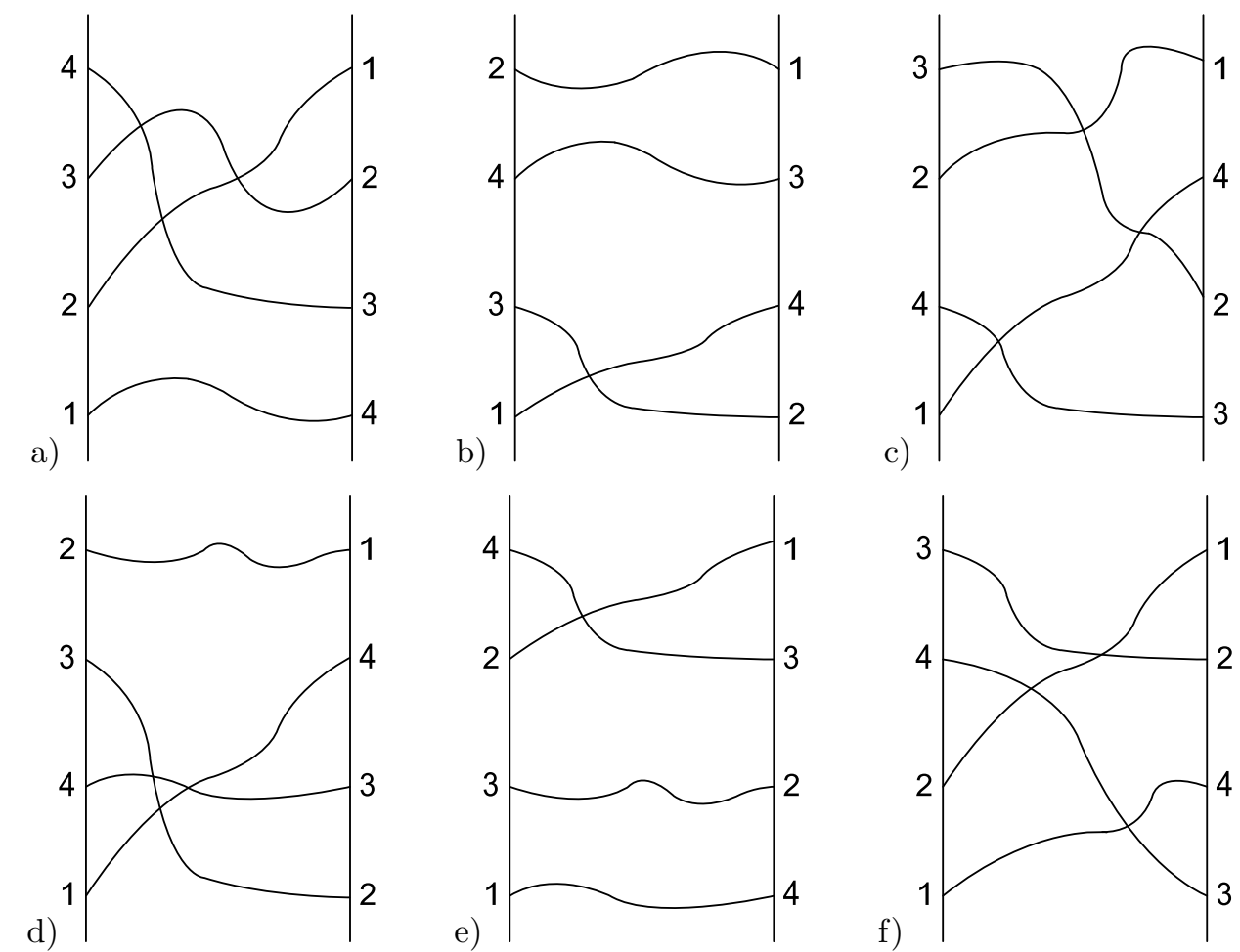

FIG. 7: All distinct level diagrams of $N=4$ maximal Hamiltonians, $H(u)=T+u V$, drawn according to (47). There are $(N-1) !=6$ distinct diagrams each corresponding to $N=4$ different orderings of eigenvalues of $V$ (see the text). For example, diagram b) corresponds to $d_{1}>d_{3}>d_{4}>d_{2}$ and three other orderings obtained with a shift of the indices by an integer $\bmod N$, e.g. $d_{2}>d_{4}>d_{1}>d_{3}$ etc. The diagrams predict either one or three level crossings in agreement with Eq. (45) and specify which levels cross. However, when the number of crossings is less than the maximum (three), additional multiple crossings of the same two levels can occur. This can increase the number of crossings by $2 K$, see Fig. 8 In the present case, the number of crossings for orderings b) and e) can increase from one to three.

$d_{1}>d_{2}>\cdots>d_{N}$, see Fig. 6. In this case Eq. (46) implies $N \rightarrow N-1$ yielding $m=N-2$ crossings, $N-1 \rightarrow N-2$ giving rise to another $m=N-3$ crossings etc., so that altogether we have $\sum_{m=1}^{N-2} m=M_{c}^{\max }$ level crossings. Note however that the schematic level diagrams, such as those shown in Figs. 4, 6, 7, and 9, do not account for the possibility of two levels crossing more than once. For example, the level $2 \rightarrow 1$ in Fig. $7 \mathrm{~b}$ can go below the level $1 \rightarrow 4$ and come back above it again generating two additional crossings, see Fig. 8. Therefore, multiple crossings of two levels can increase the total number of crossings $M_{c}$ for a given ordering of $d_{k}$ by an even number except when $M_{c}=M_{c}^{\max }$. In the latter case, since $M_{c}$ cannot exceed $M_{c}^{\max }$, multiple crossings of the same two levels are prohibited. We see that multiple crossings do not modify Eq. (45). Interestingly, numerically we have found that for as low as $N=8$, multiple crossings of the same 

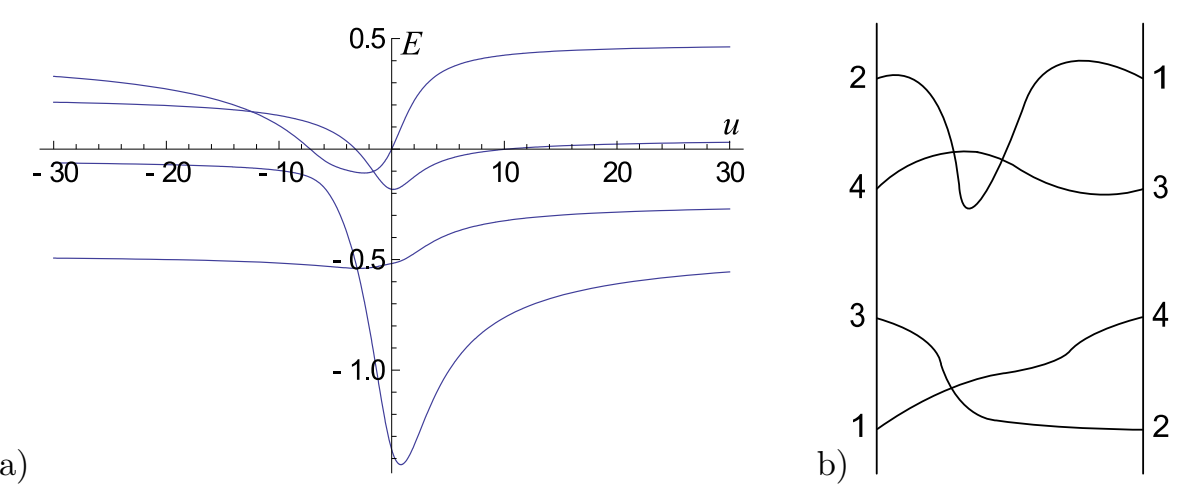

FIG. 8: a) Numerical energy levels of a $4 \times 4$ maximal Hamiltonian $H(u)=T+u V$ with the same ordering of $d_{k}$ as in Fig. 7b. Energies are scaled with $\left(u^{2}+1\right)^{-1 / 2}$ to highlight their asymptotic approach to $d_{k}$. Matrix elements of $H(u)$ are generated using Eq. (21) with random $\gamma_{k}, \varepsilon_{k}$ and random $d_{k}$ constrained to obey the above ordering. The multiple (twofold) crossing of the top two levels increases the number of crossings from one as in Fig. 7f to three. This illustrates the generic situation arising when multiple crossings increase the number of crossings by an even integer over and above the number enforced by the diagrams (47). Nevertheless, as discussed in the text, this does not affect Eq. (45). b) The schematic of a).
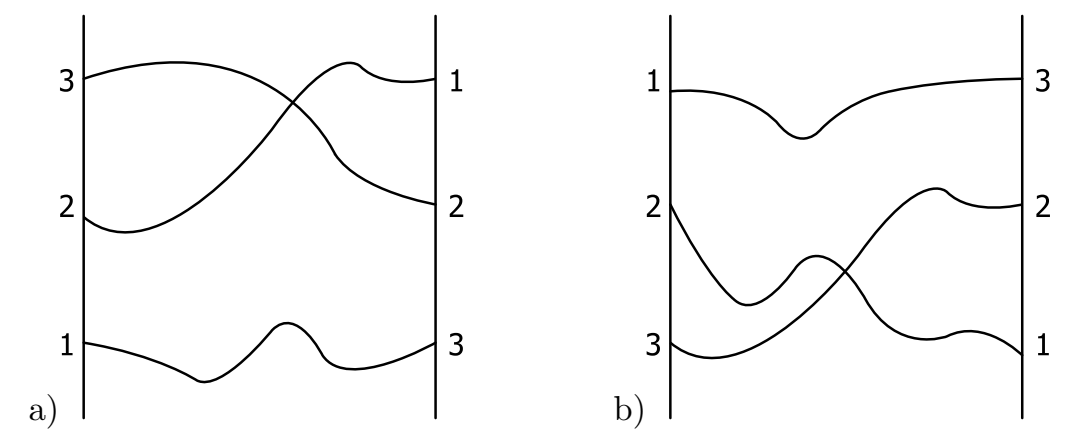

FIG. 9: All distinct level diagrams for $N=3$ maximal Hamiltonians, $H(u)=T+u V$, drawn according to (47). There are $(N-1) !=2$ distinct diagrams each corresponding to $N=3$ different orderings of eigenvalues of $V$ (see the text). For example, diagram a) corresponds to $d_{1}>d_{2}>d_{3}$ and two other orderings obtained with a shift of the indices by an integer $\bmod N$, i.e. $d_{2}>d_{3}>d_{1}$ and $d_{3}>d_{1}>d_{2}$. The diagrams predict a single level crossing in agreement with Eq. (45) and specify which levels cross. Since this is also the maximum number of crossings for $N=3$, no multiple crossings of the same two levels are allowed, cf. Fig. 7 and 8 , 
two levels are very common.

Thus far, we have established that the total number of crossings $M_{c}$ in the spectrum of an arbitrary maximally commuting Hamiltonian is $1 \leq M_{c} \leq M_{c}^{\max }$. By inspecting all level diagrams for $3 \leq N \leq 6$, we have also found that for a given $N$ the total number of level crossings changes in increments of 2 from $M_{c}^{\max }$ to 1 (2) for odd (even) $M_{c}^{\max }$, i.e. we verified Eq. (45) for these $N$. Moreover, this equation is also supported by the parity considerations in the end of Sec. III and is consistent with all preceding observations regarding the properties of level diagrams. As such, we adopt it without a formal proof.

Let us also comment that cases when some of the eigenvalues of $V, d_{k}$, are degenerate should be regarded as crossings at $u \rightarrow \pm \infty$. Equivalently, one can treat $T$ and $V$ on equal footing by defining $H(u, v)=v T+u V$. Then, degenerate $d_{k}$ correspond to crossings at $v / u=0$, while the crossings considered above occur either at finite $v / u$ or at $v / u \rightarrow \pm \infty$, or equivalently at $u / v=0$. For example, levels of the BCS Hamiltonian, which is a linear combination of Gaudin magnets [40], $\hat{H}_{B C S}=\frac{1}{B} \sum \varepsilon_{i} \hat{h}^{i}(B)+$ const, cross at the value of the BCS coupling constant $g=1 / B=1 / u=\infty$ or, equivalently, at $u=0$, see e.g. Refs. 17 and 42 .

We conclude this section with a discussion of useful properties and examples of energy level diagrams. There are $N$ ! diagrams for a given $N$ corresponding to permutations of eigenvalues $d_{1}, d_{2}, \ldots, d_{N}$. However, some of them are identical. Specifically, orderings $d_{i}<d_{m}<\cdots<d_{l}$ and $d_{i+a}<d_{m+a}<\cdots<d_{l+a}$ that differ by a shift of indices by an integer $a$ yield identical diagrams, since Eq. (46) is invariant with respect to the replacement $k \rightarrow k+a(\bmod N)$. Because $N$ different orderings can be generated using this shift, it leaves $(N-1)$ ! distinct diagrams. For example, there are two distinct diagrams for $N=3$, see Fig. 9. Each corresponds to three different orderings of $d_{k}$. Both diagrams predict a single level crossing. Since this is also the maximum number of crossings for $N=3$, repeated crossings of the same two levels are not allowed. Therefore, a single crossing of either two top or two bottom levels is the only option for $N=3$ maximally commuting Hamiltonians. For $N=4$ there are six distinct level diagrams shown in Fig. 7. Four of them diagrams a), c), d) and f) in Fig. 7 - exhibit the maximum number, $M_{c}^{\max }=3$, of level crossings. In a manner similar to that of the $N=3$ case this is the only option for the corresponding sixteen orderings of $d_{k}$. In contrast, in diagrams b) and e) showing a single crossing, multiple crossings can occur. This will increase the total number of crossings from one to three, see Fig. 8 , 


\section{SUBMAXIMAL HAMILTONIANS}

The preceding sections have focused on maximally commuting Hamiltonians, where we have explicitly constructed these operators, solved them exactly, and used the solution to explain the level crossings in such systems. In this section, we explore Hamiltonians linear in a parameter $u$ characterized by less than the maximum number of commuting partners. Most importantly, we demonstrate that some of these submaximal Hamiltonians have no energy level crossings, i.e. the inevitability of level crossings due to parameter-dependent commuting partners appears to be an exclusive property of maximal Hamiltonians.

As discussed in the Introduction, a given family of maximal Hamiltonians contains $N-1$ nontrivial independent commuting operators (see the discussion in the paragraph following Eq. (6)). It is reasonable to expect that there exist submaximal families with $N-2, N-3$ etc. Hamiltonians. Similar to Eq. (5), any common $u$-independent symmetry is assumed to be factored out by going to blocks of the same symmetry. We may adopt a convenient terminology, where families with $N-L$ nontrivial Hamiltonians are identified as being Type $L$ (cf. Type I and II of Ref. 19). Then, the maximally commuting Hamiltonians are Type 1, those with $N-2$ commuting operators are Type 2 and so on. Since a nontrivial family must contain at least two nontrivial commuting operators, the first nontrivial instance of Type 1 occurs for $N=3$, Type 2 for $N=4$ etc., where $N$ is the dimensionality of the state space.

First, let us construct $4 \times 4$ Type 2 Hamiltonians linear in a real parameter $u$. Our task is therefore to identify two $4 \times 4$ commuting real symmetric matrices that do not have the third independent commuting partner other than $(a+u b) I$. We will do so by employing the construction of maximal Hamiltonians detailed in Sec. II Consider $4 \times 4$ operators $H(x, y, u)=x T+y K+u V$ and $\widetilde{H}(x, y, u)=x \widetilde{T}+y \widetilde{K}+u \widetilde{V}$, linear in parameters $x, y$ and $u$, such that

$$
[H(x, y, u), \widetilde{H}(x, y, u)]=0 .
$$

Since this equation is to hold for all $x, y$, and $u$, the coefficients of the $x y, x u, y u$ etc. terms must vanish individually. We obtain

$$
\begin{gathered}
{[T, \widetilde{T}]=[V, \widetilde{V}]=[K, \widetilde{K}]=0,} \\
{[T, \widetilde{V}]=[\widetilde{T}, V], \quad[T, \widetilde{K}]=[\widetilde{T}, K],} \\
{[V, \widetilde{K}]=[\widetilde{V}, K] .}
\end{gathered}
$$




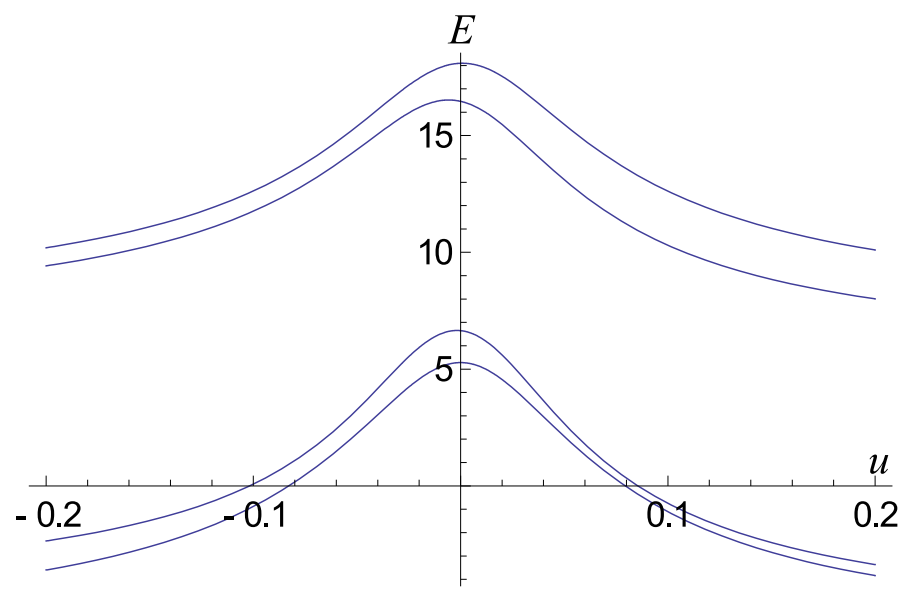

FIG. 10: Numerical energy levels of a $4 \times 4$ submaximal Hamiltonian $H(u)$ obtained from Eqs. (52) and (54) with $x_{0}=1$, random $y_{0}, d_{k}, \varepsilon_{k}$, and $\widetilde{\varepsilon}_{k}$. Energies are scaled with $\left(u^{2}+1\right)^{-1 / 2}$ as in Fig. 8. Unlike $N=4$ maximal Hamiltonians, which always have two nontrivial commuting partners, this $H(u)$ has only one such partner (see the text). Note that levels of $H(u)$ do not cross at any $u$, i.e. the mere existence of a nontrivial commuting partner does not guarantee level crossings. This is to be contrasted with $4 \times 4$ maximally commuting Hamiltonians which always exhibit either three or one crossings, see Figs. 7 and 8

Let us choose these real symmetric matrices so that $(T+u V, \widetilde{T}+u \widetilde{V})$ and $(K+u V, \widetilde{K}+u \widetilde{V})$ belong to two different families of maximally commuting Hamiltonians parameterized by $\gamma_{m}, \varepsilon_{m}$ and $\widetilde{\gamma}_{m}=\gamma_{m}, \widetilde{\varepsilon}_{m}$, respectively, see Eq. (21). Then, Eqs. (49) and (50) are satisfied by construction. Consequently, it remains to solve Eq. (51).

Thus, from Eq. (21) we have in the common eigenbasis of $V$ and $\widetilde{V}$

$$
\begin{aligned}
& {[H(x, y, u)]_{m n}=x \gamma_{m} \gamma_{n} \frac{d_{m}-d_{n}}{\varepsilon_{m}-\varepsilon_{n}}+y \gamma_{m} \gamma_{n} \frac{d_{m}-d_{n}}{\widetilde{\varepsilon}_{m}-\widetilde{\varepsilon}_{n}}, \quad m \neq n,} \\
& {[H(x, y, u)]_{m m}=u d_{m}-x \sum_{j \neq m} \gamma_{j}^{2} \frac{d_{m}-d_{j}}{\varepsilon_{m}-\varepsilon_{j}}-y \sum_{j \neq m} \widetilde{\varepsilon}_{m} \gamma_{j}^{2} \frac{d_{m}-d_{j}}{\widetilde{\varepsilon}_{m}-\widetilde{\varepsilon}_{j}},}
\end{aligned}
$$

where $d_{k}$ are the eigenvalues of $V$. Matrix elements of $\widetilde{H}(x, y, u)$ are obtained from Eq. (52) by replacing $d_{k} \rightarrow \widetilde{d}_{k}$. Using these expressions for the matrix elements, one can rewrite the remaining commutation relation (51) as follows

$$
\gamma_{l}^{2}=\frac{\left|\begin{array}{ccc}
1 & 1 & 1 \\
d_{i} & d_{j} & d_{k} \\
\widetilde{d}_{i} & \widetilde{d}_{j} & \widetilde{d}_{k}
\end{array}\right|\left|\begin{array}{ccc}
1 & 1 & 1 \\
\varepsilon_{i} & \varepsilon_{j} & \varepsilon_{k} \\
\widetilde{\varepsilon}_{i} & \widetilde{\varepsilon}_{j} & \widetilde{\varepsilon}_{k}
\end{array}\right|^{2}}{\left(\varepsilon_{i}-\varepsilon_{j}\right)\left(\varepsilon_{j}-\varepsilon_{k}\right)\left(\varepsilon_{i}-\varepsilon_{k}\right)\left(\widetilde{\varepsilon}_{i}-\widetilde{\varepsilon}_{j}\right)\left(\widetilde{\varepsilon}_{j}-\widetilde{\varepsilon}_{k}\right)\left(\widetilde{\varepsilon}_{i}-\widetilde{\varepsilon}_{k}\right)}, \quad l \neq i, j, k .
$$

Therefore, choosing $d_{r}, \widetilde{d}_{r}, \varepsilon_{r}$, and $\widetilde{\varepsilon}_{r}$, we obtain $\gamma_{r}$ from Eq. (153). This yields two commuting 
matrices $H(x, y, u)$ and $\widetilde{H}(x, y, u)$. Fixing nonzero values of $x=x_{0}$ and $y=y_{0}$, we obtain a Type 2 family of Hamiltonians linear in $u$,

$$
H(u)=\left(x_{0} T+y_{0} K\right)+u V, \quad \widetilde{H}(u)=\left(x_{0} \widetilde{T}+y_{0} \widetilde{K}\right)+u \widetilde{V}
$$

There are a number of equivalent ways to verify that these operators are indeed Type 2 rather than maximally commuting. For example, one can show that their matrix elements (52) cannot be cast into the form (21). Alternatively, it can be demonstrated that conditions (A3) necessary for any maximal operator do not hold. However, a less formal, but more fruitful verification uses the following argument. We have seen in Sec. VI that any $N=4$ maximal Hamiltonian must have either one or three level crossings. Let us check if this holds for the Hamiltonians (54). To this end, we set $x_{0}=1$, generate random $y_{0}, d_{k}, \varepsilon_{k}$, and $\widetilde{\varepsilon}_{k}$, and obtain $\gamma_{k}$ from Eq. (53) and $H(u)$ from Eq. (52). Doing so repeatedly and numerically diagonalizing the resulting Hamiltonians we observe that they always have either no or two level crossings. An example with no crossings is shown in Fig. 10, Thus, operators (54) are Type 2.

We see that level crossings are not guaranteed when the number of commuting operators is less than the maximum - nontrivial solutions of Eq. (3) do not necessarily imply crossings. The converse is also false, i.e. level crossings can occur in the absence of any nontrivial commuting partner linear in $u$ and any $u$-independent symmetry. For example, one can show that the $4 \times 4$ Hamiltonian given by Eq. (A4) in the Appendix with a single level crossing at $u=0$ has no nontrivial commuting partners and no $u$-independent symmetry. Interestingly, $N=4$ is the first dimensionality where this happens as for $3 \times 3$ real symmetric matrices linear in $u$ a level crossing implies a nontrivial commuting partner linear in $u$ and vice versa [18].

\section{SUMMARY AND OPEN QUESTIONS}

In this paper, we addressed the problem of the violation of the Wigner-von Neumann noncrossing rule in quantum integrable systems. For this purpose, we introduced and studied a general class of maximal Hamiltonians - a vector space of $N \times N$ real symmetric Hamiltonians, $H(u)=$ $T+u V$, characterized by the existence of the maximum possible number $(N)$ of independent mutually commuting integrals similarly linear in the coupling $u$. We have resolved this commutation property and explicitly constructed general maximal Hamiltonians, see Eq. (21). Interestingly, these operators are equivalent to the Gaudin magnets (30) in the next to highest weight sector, $J^{z}=J_{\max }^{z}-1$, where $J^{z}$ is the $z$ projection of the total spin. 
The mapping to Gaudin magnets allowed us to obtain a complete exact solution for the eigenstates and eigenvalues of $H(u)$, Eqs. (40) and (41). Furthermore, we have demonstrated that energy level crossings are inevitable for maximal operators, i.e. there is always at least one crossing. The total number of crossings varies from 1 or 2 to $(N-1)(N-2) / 2$, see Sec. VI. Thus, the mere existence of the maximum number of commuting partners guarantees a) an exact solution and b) level crossings. This relationship between the existence of conservation laws and exact solution is a quantum analog of the famous Liouville-Arnold theorem in classical mechanics. The latter states that if a classical model with $n$ degrees of freedom has $n$ Poisson-commuting integrals, its equations of motion are exactly solvable[20].

At the same time, by constructing an explicit example we have demonstrated that submaximal Hamiltonians - real symmetric operators of the form $T+u V$ with less than the maximum number of linear in $u$ commuting partners - often show no instances of level crossings at any $u$. Thus, the inevitability of crossings is an exclusive feature of maximally commuting operators. Similarly, we have also shown that the presence of level crossings does not necessarily imply the existence of a nontrivial commuting partner linear in $u$, i.e. crossings can occur even in the absence of such partners as well as $u$-independent symmetries.

Our understanding of properties of parameter-dependent energy spectra in integrable models is far from complete. We conclude this section with a list of open questions stemming from the results of this work.

1. We have shown that there are submaximal Hamiltonians with no level crossings. Nevertheless, crossings often do occur in these systems in violation of the non-crossing rule, see Sec. VII. This indicates that there is more to the link between crossings and the presence of commuting partners. It is interesting to understand this link for submaximal operators, what lifts level repulsion in this case, and why crossings happen only for a fraction of submaximal Hamiltonians.

2. In Sec. VII, we have also introduced a notion of Type $L$ commuting family characterized by $N-L$ nontrivial integrals. In this classification maximally commuting operators are Type 1 , while submaximal operators correspond to $L \geq 2$. A natural question is whether there is a general explicit parametrization for Type 2, 3 etc. similar to the one obtained in this paper for maximal systems. For instance, one can show that $\left|J^{z}\right| \leq J_{\max }^{z}-2$ sectors provide examples of submaximal commuting families.

3. What is the role of maximal Hamiltonians in the context of general quantum integrable 
Hamiltonians? For the central spin Hamiltonians (Gaudin magnets) they represent the next to highest weight sector. Do other integrable models have maximally commuting sectors?

4. In this paper, we focused on operators linear in the coupling $u$. An interesting question is how our results can be generalized to operators with a more general, e.g. polynomial, dependence on the coupling.

5. We have established hard bounds for the number $M_{c}$ of level crossings in $N \times N$ maximally commuting operators. Can one also determine the distribution of $M_{c}$ for large $N$, i.e. the relative prevalence of maximal Hamiltonians with a particular number of level crossings?

\section{ACKNOWLEDGEMENTS}

This research was partially supported by the National Science Foundation award NSF-DMR0547769 (H. K. Owusu and E. A. Yuzbashyan). E. A. Yuzbashyan also acknowledges the financial support by a David and Lucille Packard Foundation Fellowship for Science and Engineering, and Alfred P. Sloan Research Fellowship.

\section{APPENDIX A: SHASTRY'S CONSTRUCTION OF COMMUTING MATRICES}

In Ref. 19 Shastry developed an approach to generate commuting real symmetric $N \times N$ matrices linear in a parameter $u$. Here we show that matrices obtained with this approach belong to maximally commuting set constructed in Sec. II) see Eq. (21).

First, we briefly review the results of Ref. 19. Consider Eq. (31). In the common eigenbasis of $V$ and $\widetilde{V}$ the second relation in Eq. (3) becomes

$$
S_{i j} \equiv \frac{T_{i j}}{d_{i}-d_{j}}=\frac{\widetilde{T}_{i j}}{\widetilde{d}_{i}-\widetilde{d}_{j}}, \quad i \neq j,
$$

where $T_{i j}\left(\widetilde{T}_{i j}\right)$ are the matrix elements of $T(\widetilde{T})$ and $d_{i}$ and $\widetilde{d}_{i}$ are the eigenvalues of $V$ and $\widetilde{V}$, respectively. It remains to consider the $[T, \tilde{T}]=0$ commutation relation in Eq. (3). This can be cast into the following form:

$$
\mu_{i j k} \tilde{d}_{i}+\mu_{j k i} \tilde{d}_{j}+\mu_{k i j} \tilde{d}_{k}+\sum_{l \neq i, j, k} \nu_{l i j k} \tilde{d}_{l}=0, \quad \text { distinct } i, j, k
$$

where $\mu_{i j k}$ and $\nu_{l i j k}$ depend only on matrix elements of $H(u)$ and not on those of $\widetilde{H}(u)$. Specifically, they involve only $d_{r}, T_{r r}$ and $S_{r m}$. 
A set of particular solutions to Eq. (3) can be obtained by setting the coefficients in Eq. (A2) at each $\widetilde{d}_{r}$ individually to zero, i.e.

$$
\mu_{i j k}=0, \quad \nu_{l i j k}=0, \quad \text { distinct } l, i, j, k,
$$

Now commuting $H(u)$ and $\widetilde{H}(u)$ can be generated as follows. One first chooses $3 N-1$ parameters, e.g. $2 N-3$ variables $S_{1 r}$ and $S_{2 r}$ for $\{2,3\} \leq r \leq N$ and $N+2$ variables $\left\{d_{r}\right\}, T_{11}$, and $T_{22}$. Then, Eq. (A3) reduce to linear equations and can be solved for the remaining variables. Once $H(u)$ is determined in this way, $\widetilde{H}(u)$ can also be constructed, see Ref. 19 for details. This scheme is quite suitable for numerical implementation and, having examined several examples, Shastry observed crossings in all cases. Based on this and the results of Ref. 18 he conjectured that these matrices will always exhibit them.

To show that this construction always yields maximal Hamiltonians, we note that Eq. (A3) is a sufficient condition for Eq. (A2) to have $N$ linearly independent solutions for $\left(\widetilde{d}_{1}, \widetilde{d}_{2}, \ldots \widetilde{d}_{N}\right)$. Since $\widetilde{d}_{r}$ are the eigenvalues of $\widetilde{V}$, the existence of $N$ linearly independent solutions means that there are $N$ Hamiltonians $\widetilde{H}(u)$ with linearly independent $\widetilde{V}$ s. The absence of $u$-independent symmetries can also be demonstrated (it follows from $S_{i j} \neq 0$ for all $i \neq j$ ). Thus, we have a maximally commuting set, see the Introduction. The only difference is that by construction $d_{r}$ are not allowed to be degenerate, see Eq. (A1), while the maximal set contains these matrices as well.

Finally, we write down an example (see the discussion at the end of Sec. VII) of a $4 \times 4$ Hamiltonian $H(u)$ with a level crossing but no $u$-independent symmetry and no commuting partners linear in $u$ other than trivial ones $-c H(u)+(a+u b) I$, where $a, b$, and $c$ are real numbers and $I$ is the identity matrix,

$$
H(u)=\left(\begin{array}{cccc}
1 & 0 & 0 & 0 \\
0 & -1 & 0 & 0 \\
0 & 0 & 0 & 0 \\
0 & 0 & 0 & 0
\end{array}\right)+u\left(\begin{array}{cccc}
1 & -1 & 1 & -1 \\
-1 & 1 & -1 & 1 \\
1 & -1 & -2 & 1 \\
-1 & 1 & 1 & 2
\end{array}\right)
$$

[1] C. J. Erickson, D. Levron, W. Happer, S. Kadlecek, B. Chann, L. W. Anderson, T. G. Walker, Phys. Rev. Lett. 85, 4237 (2000).

[2] F. Haake, Quantum Signatures of Chaos (Springer, Berlin, 2006).

[3] S. Sachdev, Quantum Phase Transitions (Cambridge University Press, Cambridge, 2001).

[4] M.V. Berry and M. Wilkinson, Proc. R. Soc. A 392, 15 (1984).

[5] M. Bhattacharya and C. Raman, Phys. Rev. Lett. 97, 140405 (2006). 
[6] O. Heilman and E. H. Lieb, Trans. N.Y. Acad. Sci. 33, 116 (1970).

[7] C. S. Jain, K. Krishan, C. K. Majumdar, and V. Mubayi, Phys. Rev. B 12, 5235 (1975).

[8] T. Deguchi, K. Fabricius, and B.M. McCoy, J. Stat. Phys. 102, 701 (2001).

[9] See e.g. Fig. 1 in, E. A. Yuzbashyan, A. A. Baytin, and B. L. Altshuler, Phys. Rev. B 68, 214509 (2003).

[10] F. Hund, Z. Phys. 40, 742 (1927).

[11] J. von Neumann, E. Wigner, Z. Phys. 30, 467 (1929).

[12] E. Teller, J. Phys. Chem. 41, 109 (1937).

[13] L. D. Landau and E.M. Lifshitz, Quantum Mechanics: Non-Relativisitic Theory, pp. 304-305 (Pergamon Press, Oxford, 1980).

[14] H. C. Longuet-Higgins, Proc. R. Soc. A 344, 147 (1975).

[15] K. R. Naqvi, W. B. Brown, Int. J. Quantum Chem. 6, 271 (1972).

[16] J. P. Kestner, L.-M. Duan, Phys. Rev. A 76, 033611 (2007).

[17] M. Gaudin, Note CEA 1559, 1 (1972); J. Phys. (Paris) 37, 1087 (1976); La fonction d'onde de Bethe, (Masson, Paris, 1983). In this original work the magnetic field $B=0$ in Eq. (7). The extension to $B \neq 0$ is straightforward, see e.g. Ref. 37.

[18] E. A. Yuzbashyan, B. L. Altshuler and B. S. Shastry, J. Phys. A: Math. Gen. 35, 7525 (2002).

[19] B. S. Shastry, J. Phys. A: Math. Gen. 38431 (2005).

[20] V. I. Arnold, Mathematical Methods of Classical Mechanics (Springer-Verlag, New York, 1978).

[21] A. G. Ushveridze, Quasi-Exactly Solvable Problems in Quantum Mechanics (IOP publishing, Bristol, 1994).

[22] S. Sachdev and R. N. Bhatt, J. Appl. Phys. 61, 4366 (1987).

[23] N. V. Prokof'ev and P. C. E. Stamp, J. Phys.: Cond. Mat. 5, L663 (1993); In: Quantum Tunneling of Magnetization (ed. L. Gunther and B. Barbara), Kluwer (1995).

[24] A. V. Khaetskii, D. Loss, and L. Glazman, Phys. Rev. Lett. 88, 186802 (2002)

[25] J. Schliemann, A. Khaetskii and D. Loss, J. Phys., Condens. Matter 15, R1809 (2003).

[26] S. I. Erlingsson and Y. V. Nazarov, Phys. Rev. B 70, 205327 (2004).

[27] J. M. Taylor, A. Imamoglu, and M. D. Lukin, Phys. Rev. Lett. 91, 246802 (2003).

[28] P. Pechukas, Phys. Rev. Lett. 51, 943 (1983).

[29] We note that the restriction to Hermitian operators in the definition of the maximal set is not essential for this construction. Specifically, following the above arguments, one can show that an arbitrary maximally commuting and not necessarily Hermitian operator can be written in the form $\Sigma H(u) \Sigma^{-1}$, where $\Sigma$ is diagonal and $H(u)$ is a symmetric matrix parametrized as in Eq. (21) but with arbitrary complex parameters $\Sigma_{j j}, d_{j}, \gamma_{j}$, and $\varepsilon_{j}$.

[30] Any $u$-independent symmetry common to all $H^{i}(u)$ can always be removed by going to smaller blocks.

[31] H. Baumgrtel, Analytic perturbation theory for matrices and operators, pp. 104-106, (Birkhuser Verlag, Basel, 1985).

[32] This can be seen by evaluating $m n^{\text {th }}$ matrix elements of $\sum_{k=1}^{N} f_{k}(u) h^{k}(u)=0$ for $m \neq n$ and using 
Eqs. (19) and (20).

[33] E. K. Sklyanin, J. Sov. Math. 47, 2473 (1989).

[34] E. K. Sklyanin, Progr. Theoret. Phys. Suppl. 118, 35 (1995).

[35] L. Amico, G. Falci, R. Fazio, J. Phys. A 34, 6425 (2001).

[36] Jan von Delft and R. Poghossian, Phys. Rev. B 66, 134502 (2002).

[37] J. Dukelsky, S. Pittel, G. Sierra, Rev. Mod. Phys. 76643 (2004).

[38] J. Bardeen, L.N. Cooper, and J.R. Schriefer, Phys. Rev. 1081175 (1957).

[39] R. W. Richardson and N. Sherman, Nucl. Phys. 52, 221 (1964); 52, 253 (1964).

[40] M. C. Cambiaggio, A. M. F. Rivas, and M. Saraceno, Nucl. Phys. A 624, 157 (1997).

[41] F. A. de la Cruz and A. I. Nesterov, in: Non-Associative Algebra and its Applications (ed. L. Sabinin, L. Sbitneva, and I. Shestakov), Lect. Notes Pure Appl. Math. 246, Chapman \& Hall/CRC Press, (2006).

[42] J. M. Roman, G. Sierra, and J. Dukelsky, Phys. Rev. B 67, 064510 (2003). 